\title{
Vitronectin Binds to a Specific Stretch within the Head Region of Yersinia Adhesin A and Thereby Modulates Yersinia enterocolitica Host Interaction
}

\author{
Melanie C. Mühlenkamp ${ }^{a}$ Teresia Hallström ${ }^{b}$ Ingo B. Autenrieth ${ }^{a}$ \\ Erwin Bohn $^{a}$ Dirk Linke ${ }^{e}$ Janina Rinker ${ }^{a}$ Kristian Riesbeck $^{f} \quad$ Birendra Singh $^{d}$ \\ Jack C. Leo ${ }^{\text {e Sven Hammerschmidt }}{ }^{d}$ Peter F. Zipfel ${ }^{b, c}$ Monika S. Schütz ${ }^{a}$ \\ a Institute for Medical Microbiology and Hygiene, University Hospital Tübingen, Tübingen, ${ }^{b}$ Department of \\ Infection Biology, Leibniz Institute for Natural Product Research and Infection Biology, Hans Knöll Institute, and \\ ${ }^{c}$ Friedrich Schiller University Jena, Jena, and d Department of Microorganism Genetics, Interfaculty Institute

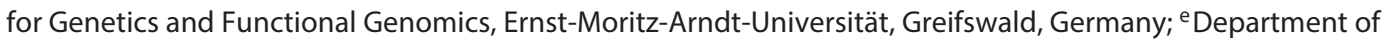 \\ Biosciences, University of Oslo, Oslo, Norway; ${ }^{f}$ Clinical Microbiology, Department of Translational Medicine, \\ Lund University, Malmö, Sweden
}

\section{Key Words}

Bacterial infection - Cell surface molecules - Complement .

Yersinia adhesin A - Vitronectin

\begin{abstract}
Complement resistance is an important virulence trait of Yersinia enterocolitica $(\mathrm{Ye})$. The predominant virulence factor expressed by $\mathrm{Ye}$ is Yersinia adhesin $\mathrm{A}(\mathrm{YadA})$, which enables bacterial attachment to host cells and extracellular matrix and additionally allows the acquisition of soluble serum factors. The serum glycoprotein vitronectin $(\mathrm{Vn})$ acts as an inhibitory regulator of the terminal complement complex by inhibiting the lytic pore formation. Here, we show YadA-mediated direct interaction of $Y e$ with $V n$ and investigated the role of this $\mathrm{V} n$ binding during mouse infection in vivo. Using different Yersinia strains, we identified a short stretch in the YadA head domain of Ye O:9 E40, similar to the 'uptake region' of Y. pseudotuberculosis YPIII YadA, as crucial for efficient $\mathrm{Vn}$ binding. Using recombinant fragments of $\mathrm{Vn}$, we found the C-terminal part of $\mathrm{Vn}$, including heparin-binding
\end{abstract}

domain 3, to be responsible for binding to YadA. Moreover, we found that $\mathrm{Vn}$ bound to the bacterial surface is still functionally active and thus inhibits C 5 b- 9 formation. In a mouse infection model, we demonstrate that $\mathrm{Vn}$ reduces complement-mediated killing of Ye O:9 E40 and, thus, improved bacterial survival. Taken together, these findings show that YadA-mediated $\mathrm{V} n$ binding influences Ye pathogenesis.

(c) 2016 S. Karger AG, Basel

\section{Introduction}

Yersinia enterocolitica (Ye) and Yersinia pseudotuberculosis (Yps) are enteropathogens causing enteric and systemic diseases $[1,2]$. Besides the chromosomally encoded adhesins invasin (Inv) and Ail [3-5], the trimeric autotransporter adhesin (TAA) Yersinia adhesin A (YadA) is the decisive factor that determines the pathogenicity of $Y e$ [6]. YadA forms rigid fibrous structures, which protrude approximately $23 \mathrm{~nm}$ from the cell surface $[7,8]$, and mediates adhesion to extracellular matrix (ECM) pro-

\section{KARGER}

(c) 2016 S. Karger AG, Basel

E-Mail karger@karger.com

www.karger.com/jin
Dr. Monika S. Schütz

Institute for Medical Microbiology and Hygiene, University Hospital Tübingen Elfriede-Aulhorn-Strasse 6

DE-72076 Tübingen (Germany)

E-Mail monika.schuetz@med.uni-tuebingen.de 
teins such as collagen, fibronectin and laminin and also to complement factors [9]. Being the prototype of the TAA family of proteins, YadA is characterized by a modular composition of several domains; the extracellularly located N-terminal head domain is followed by a connector element (also called the neck region) leading into a coiledcoil stalk. The stalk is connected to the C-terminal translocator or membrane anchor domain, consisting of 4 $\beta$-strands per monomer [9]. To form a functional adhesin on the bacterial surface, 3 YadA monomers trimerize and form the pore of the translocator domain, which is inserted into the outer membrane [10]. The translocator enables the transport of the passenger domains onto the bacterial surface, where they also form obligate trimers [9].

YadA knockout strains of $Y e$ are avirulent and do not cause infection in a mouse model [11-13]. This striking effect has been attributed mainly to the reduced efficiency of effector protein (Yop) delivery by a dedicated type 3 secretion system (T3SS) which requires proper adhesion to host cells; loss of adherence results in the inability to resist phagocytosis $[14,15]$. However, in $Y p s$, which is more closely related to Yersinia pestis, YadA is dispensable for virulence and Yop injection [16]. YadA of Yps and $Y e$ not only differ in their role during infection, but also in the sequence and binding repertoire of host ECM proteins and cellular receptors. YadA of Yps carries an additional stretch within its head region that enables entry into host cells [17]. This important stretch is absent in YadA of several $Y e$ serotypes and strains. Moreover, the binding capacities of YadA differ between $Y e$, which binds collagen and laminin, and Yps, which binds fibronectin [18].

By interacting with several complement factors, serum resistance is an important virulence trait of $Y e$. It has been shown that factor $\mathrm{H}, \mathrm{C} 4 \mathrm{~b}$-binding protein $(\mathrm{C} 4 \mathrm{BP})$ and $\mathrm{C} 3$ bind to the YadA stalk domain and thus inhibit complement killing $[19,20]$. Recently, we demonstrated a novel mechanism that contributes to serum resistance in $Y e \mathrm{O}: 8$ WA-314, and amended the current model of direct factor $\mathrm{H}$ binding to $\mathrm{YadA}^{0: 3}$ and $\mathrm{YadA}^{0: 9}$. We have shown that $Y e$ binds $\mathrm{C} 3 \mathrm{~b}$ or $\mathrm{iC} 3 \mathrm{~b}$ and thereby attracts high amounts of factor $\mathrm{H}$ to the bacterial surface [21]. This is different from the direct binding of factor $\mathrm{H}$, which was shown earlier $[19,20,22]$. Importantly, by binding these complement regulatory factors, $\mathrm{Ye}$ is able to interfere with complement activity by inhibiting complement-mediated killing at an early stage of the cascade.

The human glycoprotein Vn is synthesized in the liver and secreted into plasma [23], where it is present as a monomer (65 and $75 \mathrm{kDa})$ at high concentrations (200-
$400 \mu \mathrm{g} / \mathrm{ml}$ ) [24]. Vn also exists as an extravascular cellbound multimeric form in several tissues, and Vn mRNA can be detected in high concentrations in the liver, brain, heart and adipose tissue but is rare or absent in the kidney and spleen [25]. It comprises an $\mathrm{N}$-terminal somatomedin-binding domain, consisting of 43 amino acid (aa) residues, followed by the host cell integrin receptorbinding motif RGD (Arg-Gly-Asp). In addition to 4 hemopexin-like domains with unknown function, $\mathrm{Vn}$ also contains 3 heparin-binding domains (HBDs) which span aa 82-137 (HBD-1), aa 175-219 (HBD-2) and aa 348361 (HBD-3) [26, 27]. Vn is an important regulator of complement activity at the level of terminal complement complex (TCC) formation and a component of the ECM, and it also fulfills functions in cell migration and tissue repair [27].

At the level of TCC formation, Vn regulates complement activity by directly binding to the protein complex C5b-7 or to C9 [28]. The exact mode of regulation is not fully understood. It has been postulated, however, that $\mathrm{Vn}$ binds the nascent precursor complex C5b-7, resulting in a Vn-C5b-7 complex that is unable to insert into the cell membrane $[27,28]$. Vn can also directly bind C9 and thereby inhibit C9 polymerization. This binding takes places through HBD-3 whereas the binding site for the nascent C5b-7 is still unknown [27-29].

A wide variety of bacteria bind Vn via various surface proteins. The respiratory pathogens Moraxella catarrhalis $(\mathrm{Mc})$ and Haemophilus influenzae (Hi) as well as the urogenital pathogen Haemophilus ducreyi express proteins belonging to the TAA family. These proteins are the ubiquitous surface protein A2 (UspA2) of Mc, the Haemophilus surface fibrils (Hsf) and the Haemophilus adhesin (Hia) of $\mathrm{Hi}$ or the $\mathrm{H}$. ducreyi serum resistance protein A (DsrA) [9, 30-36]. In the invasive bacterial pathogen Neisseria meningitidis the 3 proteins Opc, Opa and Msf interact with Vn [37-40]. However, to date, no enteropathogenic bacteria have been reported to use Vn to escape complement-mediated attack and thus mediate serum resistance.

Ye has evolved a multitude of mechanisms to evade the host immune system. Amongst these, serum resistance is of uttermost importance. The significance of the complement regulator $\mathrm{Vn}$ in complement evasion and modulation of host cell interaction with bacterial and fungal pathogens has recently been recognized $[27,30-32,37$, $39-44]$. $Y e$ is able to bind several regulators of complement activity. The role of $\mathrm{Vn}$ in $Y e$ host cell interaction and in pathogenicity has not yet been addressed in detail, but it was shown in previous studies that YadA from $Y e$
Mühlenkamp et al. 
Table 1. Plasmids used in this study

\begin{tabular}{llcc}
\hline Plasmid name & Description & Resistance & Reference \\
\hline pBla & $\begin{array}{l}\text { expression of YopE aa 1-53 } \beta \text {-lactamase hybrid protein under control } \\
\text { of the YopE promoter }\end{array}$ & kanamycin & 46 \\
\hline pACYC184 EGFP & EGFP expressed under control of a constitutive tac/lac promoter & chloramphenicol & 47 \\
\hline pASK-IBA4C_yadAO:8 & $\begin{array}{l}\text { yadA from Ye O:8 WA-314 cloned into pASK-IBA4C; expression under } \\
\text { control of an anhydrotetracycline-inducible promoter }\end{array}$ & chloramphenicol this study \\
\hline pASK-IBA4C_yadAO:9 & $\begin{array}{l}\text { yadA from Ye O:9 E40 cloned into pASK-IBA4C; expression under } \\
\text { control of an anhydrotetracycline-inducible promoter }\end{array}$ & chloramphenicol & this study \\
\hline $\begin{array}{l}\text { pASK-IBA4C_yadAO:9/ } \\
\text { O:8 hybrid }\end{array}$ & $\begin{array}{l}\text { plasmid for inducible expression of a hybrid protein consisting of the } \\
\text { yadA from Ye O:8 WA-314; expression under control of an } \\
\text { anhydrotetracycline-inducible promoter }\end{array}$ & chloramphenicol & this study \\
\hline $\begin{array}{l}\text { pASK-IBA4C_yadAO:9 } \\
\text { puptake region }\end{array}$ & $\begin{array}{l}\text { plasmid for inducible expression of yadA from Ye O:9 E40 lacking aa } \\
\text { anhydrotetracycline-inducible promoter }\end{array}$ & chloramphenicol & this study \\
\hline
\end{tabular}

O:8 does not bind Vn under stringent assay conditions [45]. In this study, we systematically investigated (1) Vn binding of different $Y e$ strains, (2) which components of $Y e$ might enable this binding and (3) how this interaction modulates $Y e$ serum resistance, host cell interaction and overall pathogenicity. Importantly, we were able to demonstrate a novel mechanism that facilitates Ye serum resistance mediated by the surface adhesin YadA binding to Vn. We found that subtle differences within the YadA head domain of different Yersinia strains determine the efficacy of the Vn binding. An additional stretch in $Y e$ $\mathrm{YadA}^{\mathrm{O}: 9}$, which is similar to the 'uptake region' of Yps YadA $^{\text {YPIII }}$ [18], was identified as a crucial region for the high-affinity binding of Vn. Moreover, we located HBD3 within $\mathrm{Vn}$ as the YadA binding site. Notably, bound Vn is active on the bacterial surface and protects bacteria from complement-mediated lysis by the inhibition of C9 polymerization. This mechanism allows the enhanced survival of Ye O:9 E40 during the early phase of a mouse infection in vivo.

\section{Materials and Methods}

\section{Mice}

C57BL/6 wild-type (WT) mice were purchased from Harlan Winkelmann (Horst, The Netherlands). B6.129S2(D2)-Vtn ${ }^{t m 1 D g i} / \mathrm{J}$ mice (http://jaxmice.jax.org/strain/004371.html) with a C57BL/6 background were purchased from Jackson Laboratories (Bar Harbor, Maine, USA). All mice were bred under specific pathogen-

YadA-Mediated Interaction of $Y e$ with Vitronectin free conditions in individually ventilated cages with access to water and food ad libitum. Experiments were performed with female mice (aged 6-8 weeks) according to German law with the permission of the Regierungspräsidium Tübingen (permission No. $\mathrm{H} 4 / 15)$.

\section{Plasmids}

Plasmids used in this study are listed in table 1.

\section{Bacterial Strains and Culture Conditions}

All Yersinia strains were cultivated in lysogeny broth medium with supplements (antibiotics as listed in table 2) overnight at $27^{\circ} \mathrm{C}$. To promote YadA expression, a 1:20 dilution of the overnight culture was made with fresh medium and incubated for $3 \mathrm{~h}$ at $37^{\circ} \mathrm{C}$. Moraxella strains were grown overnight at $37^{\circ} \mathrm{C}$ in brainheart infusion medium. All bacteria were washed twice with PBS, and the optical density at $600 \mathrm{~nm}$ was determined. The number of bacteria used for the individual experimental setups are indicated in the respective sections. All bacterial strains used in this study are listed in table 2.

\section{Serum}

Normal human serum (NHS) was collected from at least 4 healthy volunteers and pooled. Aliquots were stored at $-80^{\circ} \mathrm{C}$ and thawed only once. Heat-inactivated serum (HIS) was generated by incubation at $56^{\circ} \mathrm{C}$ for $30 \mathrm{~min}$ immediately before use.

\section{Antibodies}

Antibodies used in this study are listed in table 3.

Purified Proteins Used in This Study

Purified monomeric and multimeric Vn was purchased from BD Bioscience (Heidelberg, Germany) and Millipore (Schwalbach, Germany), respectively. Vn fragments were expressed and purified as described previously $[35,54]$. 
Table 2. Bacterial strains used in this study

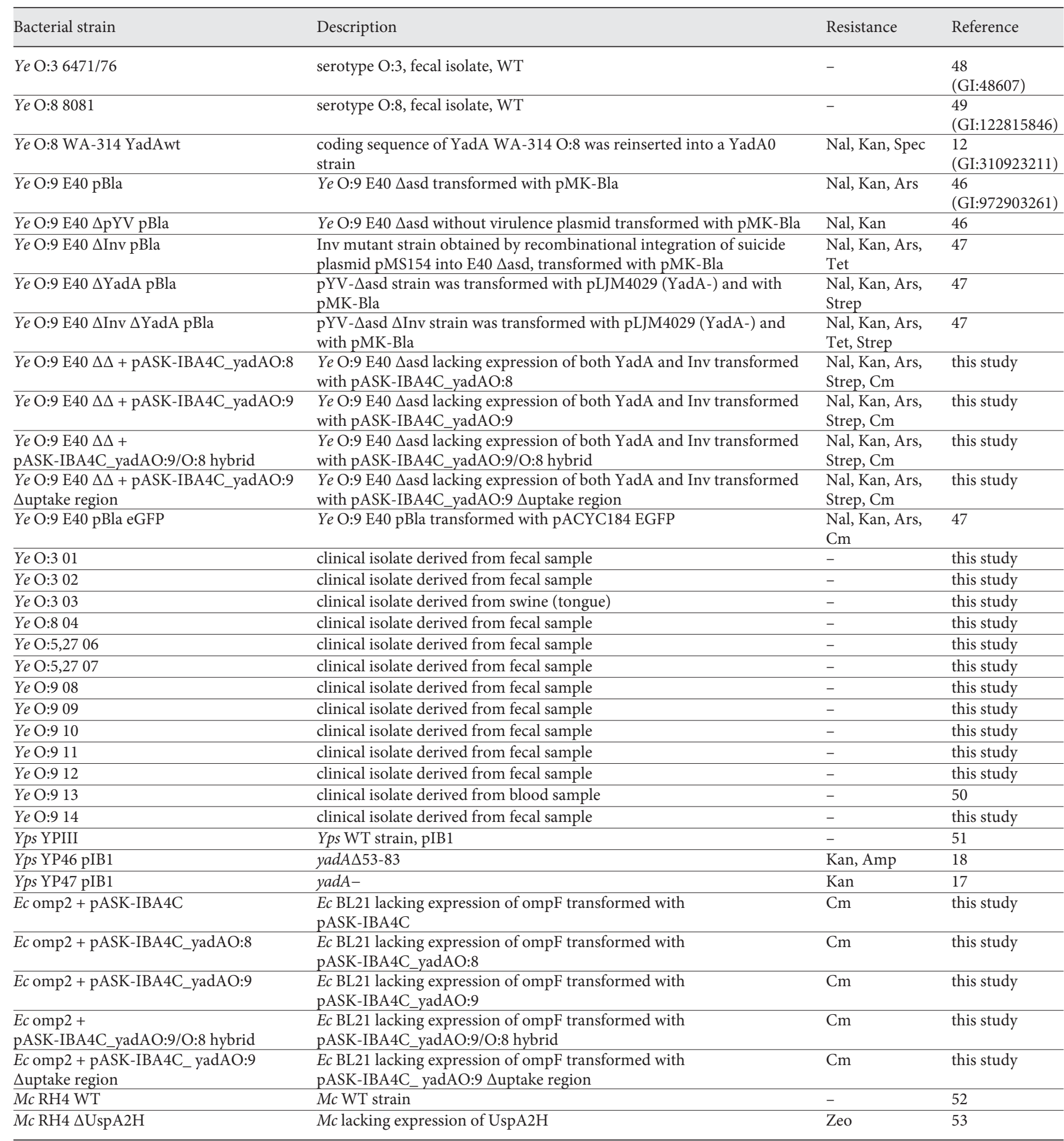

Amp = Ampicillin; Ars = arsenite $\mathrm{Cm}=$ chloramphenicol; $\mathrm{Kan}=$ kanamycin; Nal = nalidixic acid; Spec = spectinomycin; Strep = streptomycin; Tet = tetracycline; $\mathrm{Zeo}=$ zeocine. 
Table 3. Antibodies used in this study

\begin{tabular}{|c|c|c|c|c|}
\hline & Conjugate & Clone & Manufacturer & $\begin{array}{l}\text { Working } \\
\text { dilutions }\end{array}$ \\
\hline \multicolumn{5}{|l|}{ Primary antibodies } \\
\hline Goat anti-factor $\mathrm{H}$ & - & polyclonal & Complement Technology & $1: 100$ \\
\hline Rabbit anti-Vn & - & polyclonal & Complement Technology & $\begin{array}{l}\text { FACS 1:100; } \\
\text { WB 1:1,000 }\end{array}$ \\
\hline Rabbit anti-Ye YadA & - & polyclonal & Lab antibody; I. Autenrieth & $1: 200$ \\
\hline Rabbit anti-Yps YadA & - & polyclonal & Lab antibody; P. Dersch & $1: 200$ \\
\hline Sheep anti-Vn & - & polyclonal & AbD Serotech & $1: 100$ \\
\hline Mouse anti-human C5b-9 & - & aE11 & Dako & $1: 1,000$ \\
\hline \multicolumn{5}{|l|}{ Mouse anti- $\beta$ subunit of } \\
\hline E.coli RNA-polymerase & - & 8RB13 & NeoClone Biotechnology & $1: 2,000$ \\
\hline \multicolumn{5}{|l|}{ Secondary antibodies } \\
\hline Donkey anti-rabbit & APC & & Jackson ImmunoResearch & $1: 200$ \\
\hline Goat anti-rabbit & DyLight 800 & & Thermo Scientific & $1: 10,000$ \\
\hline Goat anti-rabbit & DyLight 680 & & Thermo Scientific & $1: 10,000$ \\
\hline Goat anti-mouse & DyLight 680 & & Thermo Scientific & $1: 10,000$ \\
\hline Rabbit anti-sheep & DyLight 800 & & Thermo Scientific & $1: 10,000$ \\
\hline Rabbit anti-goat & Alexa Fluor 488 & & Jackson ImmunoResearch & $1: 200$ \\
\hline Goat anti-mouse & Alexa Fluor 647 & polyclonal & Jackson ImmunoResearch & $1: 2,500$ \\
\hline
\end{tabular}

Binding Assay with Serum or Purified Proteins Analyzed by Flow Cytometry

To analyze the binding of purified $\mathrm{Vn}$ or $\mathrm{Vn}$ and factor $\mathrm{H}$ from HIS, a total of $1 \times 10^{7}$ bacteria per assay were incubated with $5-50 \%$ HIS or purified Vn $(1-10 \mu \mathrm{g} / \mathrm{ml})$ diluted with PBS (Life Technologies, Darmstadt, Germany) in a total volume of $100 \mu \mathrm{l}$ for $30 \mathrm{~min}$ at $37^{\circ} \mathrm{C}$. As an internal control, each strain was also treated with PBS only. Recombinant Vn fragments were used at $4 \mu \mathrm{g} / \mathrm{ml}$. After washing with $1 \%$ BSA in PBS (washing buffer), bacteria were spun down and the pellet was resuspended in $200 \mu \mathrm{l} 4 \%$ paraformaldehyde in PBS for $1 \mathrm{~h}$ at room temperature. Bacteria were washed once again and finally incubated with primary polyclonal antibodies (pAb) directed against $\mathrm{Vn}$ or factor $\mathrm{H}$ overnight at $4^{\circ} \mathrm{C}$. The next day, bacteria were washed once and incubated with suitable secondary antibodies for $1 \mathrm{~h}$ at room temperature. After a final washing step, bacteria were transferred to FACS tubes and analyzed with a Fortessa LSR II instrument. Data analysis was carried out using WinMDI v2.8. The PBS-only control was used to determine background staining using the same primary and secondary antibodies as for all other samples. Values obtained for the control samples were subtracted from the values obtained for the corresponding samples that were incubated in serum or purified Vn. All flow cytometry figures show background subtracted values.

Detection of Vn Binding or YadA Expression by Western Blot

To analyze Vn binding by immunoblotting, $5 \times 10^{8}$ bacteria (bacterial numbers were determined photometrically by measuring the optical density at $600 \mathrm{~nm}$; a volume corresponding to the desired number of bacteria was harvested by centrifugation, and the bacterial pellets were then used to carry out the assay) were incubated in $100 \mu$ of $50 \%$ HIS diluted in PBS as described above. Thereafter, bacteria were washed twice with washing buffer, once with PBS, and finally resuspended in $50 \mu \mathrm{l}$ deionized water. For the detection of YadA, bacteria were simply washed after harvest. After the addition of $25 \mu \mathrm{l} 4 \times$ Laemmli buffer (Bio-Rad Laboratories, Munich, Germany), samples were boiled for $5 \mathrm{~min}$ at $95^{\circ} \mathrm{C}$ and separated in a $10 \%$ acrylamide SDS gel (Bio-Rad Laboratories). Each lane was loaded with an equal number of bacteria. After blotting, the membranes were blocked with 3\% BSA and 5\% milk powder in TBS for $1 \mathrm{~h}$ at room temperature. They were then incubated with the desired antibodies (a complete list of antibodies and working dilutions is given in table 3 ) for $1 \mathrm{~h}$ at room temperature or at $4^{\circ} \mathrm{C}$ overnight, washed with $0.1 \%$ TBS-T and then incubated with the suitable secondary antibody. Fluorescence signals were recorded using a LICOR Odyssey imaging system.

Detection of Vn Binding by Blot Overlay Assay

Bacterial lysates were prepared as described above, separated by SDS-PAGE and blotted. After blocking with 5\% milk, 3\% BSA in PBS for $3 \mathrm{~h}$ at room temperature, the membrane was incubated with $7 \mu \mathrm{g} / \mathrm{ml}$ purified monomeric Vn in 3\% BSA in PBS-T overnight at $4^{\circ} \mathrm{C}$. After washing with $0.1 \%$ TBST, Vn was detected with rabbit anti-Vn pAb and a secondary DyLight 680-conjugated goat anti-rabbit pAb. Fluorescence signals were recorded using a LICOR Odyssey imaging system.

\section{Purification of DNA from Yersinia Colonies}

Yersinia strains were streaked on the LB agar plates without antibiotics. The next day, a single colony was used for DNA extraction using the Qiagen QIAmp DNA mini kit according to the manufacturer's protocol. DNA was finally eluted in $100 \mu$ l of ultrapure water. 
PCR Amplification of the YadA Head Region

To test Yersinia YadA for the presence of the additional stretch (enabling the recruitment of $\mathrm{Vn}$ ) within its head region, we used the primers YadA_Seroseq_435F ( $5^{\prime}$-gatcagtgtctctgcggcat- $\left.3^{\prime}\right)$ and YadA_Seroseq_435R ( $5^{\prime}$-gccccataagtaactgccga- $\left.3^{\prime}\right)$ that bind to highly conserved regions upstream and downstream of the uptake region (online suppl. fig. S1; for all online suppl. material, see www. karger.com/doi/10.1159/000449200). According to the sequence alignment, the PCR reaction should yield a fragment of $442 \mathrm{bp}$ with Ye O:9 E40 or 451 bp with Yps YPIII (both harboring the uptake region of approx. $90 \mathrm{bp}$ ) or $337 \mathrm{bp}$ with $\mathrm{Ye}$ O:8 WA-314 and 346 bp with $Y e$ O:3 6471/76 and $Y e$ O:5.27 (all 3 lacking the uptake region) and thus allow us to discriminate between YadA with and without the uptake region. We used the following PCR program: $2 \min 95^{\circ} \mathrm{C}$ (initial denaturation), $30 \mathrm{~s} 95^{\circ} \mathrm{C} \rightarrow 1 \min 55^{\circ} \mathrm{C} \rightarrow$ $30 \mathrm{~s} 68^{\circ} \mathrm{C}$ (repeated 29 times), $5 \mathrm{~min} 72^{\circ} \mathrm{C}$ (final extension) and cooling at $4^{\circ} \mathrm{C}$ until further processing.

Separation of PCR Products by Capillary Gel Electrophoresis

To determine the size of the PCR products, they were analyzed using a QIAxcel capillary gel electrophoresis system according to the manufacturer's protocol.

\section{DNA Sequencing}

PCR products were purified using the Promega Wizard ${ }^{\circledR}$ SV gel and PCR Clean-Up System according to the manufacturer's protocol. Subsequently, Sanger sequencing was performed by GATC using the same primers as for the PCR reaction.

\section{Heparin Inhibition Assay}

Sterile glass coverslips were coated with purified $\mathrm{Vn}(10 \mu \mathrm{g} / \mathrm{ml})$ at $4^{\circ} \mathrm{C}$ overnight and air-dried. The coverslips were then placed in a 24-well plate and either incubated with PBS or $100 \mu \mathrm{M}$ heparin in PBS; $5 \times 10^{7}$ bacteria ( $Y e$ O:9 E40 pBla EGFP) were added to each well, spun down for 5 min at $300 \mathrm{~g}$ and incubated for $1 \mathrm{~h}$ at $37^{\circ} \mathrm{C}$ in a humidified atmosphere. Afterwards, the supernatant was removed, and the samples were washed 2 times and finally fixed by the addition of $4 \%$ paraformaldehyde in PBS. After washing, coverslips were mounted in Mowiol, and micrograph pictures were acquired using a Zeiss LSM 510. To quantify adhesion, the number of bacteria for a given field of view (representative for the entire coverslip) was counted.

\section{Analysis of C5b-9 Deposition by Flow Cytometry}

To analyze whether bound $\mathrm{Vn}$ was functionally active, bacteria were incubated with $\mathrm{Vn}(10-50 \mu \mathrm{g} / \mathrm{ml})$ or C4BP $(10-50 \mu \mathrm{g} / \mathrm{ml})$ for $30 \mathrm{~min}$ at $37^{\circ} \mathrm{C}$. After washing, bacteria were incubated with C5b-6 $(1 \mu \mathrm{g} / \mathrm{ml})$ and C7 $(1 \mu \mathrm{g} / \mathrm{ml})$ for $10 \mathrm{~min}$, and then C8 $(0.4 \mu \mathrm{g} /$ $\mathrm{ml})$ and $\mathrm{C} 9(1 \mu \mathrm{g} / \mathrm{ml})$ were added for $30 \mathrm{~min}$ at $37^{\circ} \mathrm{C}$. All complement components except for $\mathrm{Vn}$ were from Complement Technology (Tyler, Tex., USA). Deposited C5b-9 was detected by mouse anti-human C5b-9 mAb followed by Alexa Fluor 647-conjugated goat anti-mouse pAb. After 2 additional washes, bacteria were analyzed by flow cytometry (EPICS XL-MCL; Coulter, Hialeah, Fla., USA). All incubations were kept in a final volume of $100 \mu \mathrm{l} 1 \% \mathrm{BSA}$ in PBS, and washes were performed with the same buffer. Primary and secondary pAb were added separately as negative controls for each strain analyzed.
In vitro Serum Killing Assay

To analyze the susceptibility of $Y e$ and Yps to complementmediated killing in human serum, $5 \times 10^{6}$ bacteria were incubated in $100 \mu \mathrm{l} 20 \% \mathrm{NHS}$ or HIS for $30 \mathrm{~min}$ at $37^{\circ} \mathrm{C}$. Complement activity was stopped by adding $100 \mu \mathrm{BHI}$ medium and placing the samples for 5 min on ice. Afterwards, serial dilutions of the samples were prepared, plated on selective agar plates and incubated at $27^{\circ} \mathrm{C}$ for $48 \mathrm{~h}$. The colony-forming units (CFU) were determined. The serum bactericidal effect was calculated as the survival percentage, taking the bacterial counts obtained with bacteria incubated in HIS as $100 \%$.

\section{In vivo Serum Killing Assay}

To analyze the lytic activity of serum complement against $Y e$ in $\mathrm{C} 57 \mathrm{BL} / 6$ and B6.129S2(D2)-Vtn ${ }^{t m 1 D g i} / \mathrm{J}$ mice, the animals were infected intravenously with $1 \times 10^{7}$ bacteria. After $30 \mathrm{~min}$, they were sacrificed by $\mathrm{CO}_{2}$ asphyxiation and blood was withdrawn from the heart. Heparin $(100 \mu \mathrm{l}$ at $100 \mu \mathrm{g} / \mathrm{ml})$ (Sigma-Aldrich, Steinheim, Germany) was mixed with the blood to avoid coagulation. Serial dilutions of the samples were plated on selective agar and incubated at $27^{\circ} \mathrm{C}$ for $48 \mathrm{~h}$. The CFU were determined by counting the colonies.

\section{Bioinformatics and Statistical Analysis}

The GI numbers or the references of the sequences used in this work are listed in table 2. Alignments were produced with Kalign or Muscle and further edited manually $[55,56]$. Data are expressed as means \pm SD and were analyzed with the Student $t$ test or with one-way ANOVA for multiple comparisons as described in the figure legends. GraphPad Prism v6.0 was used to analyze the data (GraphPad Software, La Jolla, Calif., USA). Differences were considered significant if $\mathrm{p} \leq 0.05$.

\section{Results}

\section{Ye O:9 E40 Efficiently Binds Vn}

$\mathrm{Vn}$ plays an important role in the complement resistance of, for example, $\mathrm{Mc}, \mathrm{Hi}$ and Streptococcus pneumoniae $[32,34,44,54,57]$. In order to test if $Y e$ is able to bind $\mathrm{Vn}$ and if there are differences in the binding capacity of various $Y e$ strains and serotypes, we incubated a set of strains in 50\% HIS, washed the cells and detected Vn bound to bacteria by immunostaining with antibodies directed against $\mathrm{Vn}$ and subsequent flow cytometry analysis (fig. 1a). Upon incubation with HIS, we found very diverse binding properties of $Y e$ strains compared to $M c$ RH4 and Yps YPIII. Mc RH4 served as a positive control $[30,34]$, whereas Yps YPIII was used as an additional comparator. It has been recognized that Yps YPIII YadA differs from the YadA sequences of other strains and that this difference coincides with a change in preferred ECM binding partners; this might possibly also affect the interaction with Vn (Yps YPIII YadA preferentially binds to fibronectin instead of collagen and laminin as observed 


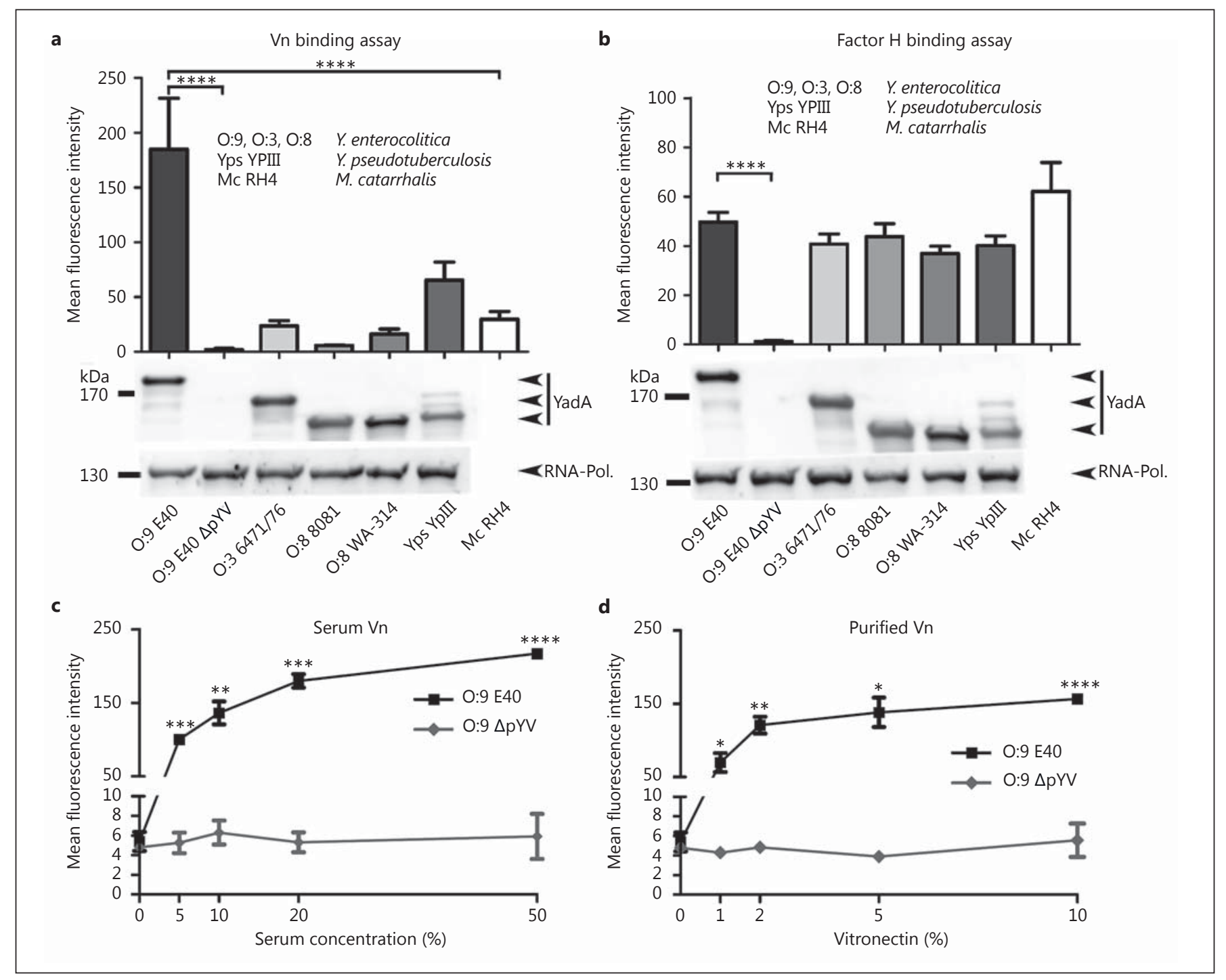

Fig. 1. Vn is efficiently bound by Ye O:9 E40 and Yps. a Several strains of $Y e$, serotype $0: 9$ with and without virulence plasmid (O:9 E40 and O:9 E40 $\Delta \mathrm{pYV}$ ), serotype O:3 (O:3 6471/76) and serotype O:8 (O:8 8081; O:8 WA-314), and 1 Yps (Yps YPIII) WT strain were incubated with HIS, washed and subsequently analyzed for the presence of $\mathrm{Vn}$ on the bacterial surface by flow cytometry. Mc (Mc RH4), which is known to bind $\mathrm{Vn}$ and Yps, which we supposed also binds $\mathrm{Vn}$, were included as a positive control for $\mathrm{Vn}$ binding. Ye O:9 E40, cured from the virulence plasmid (plasmid of Yersinia virulence; $\mathrm{pYV}$ ) that encodes for the Ye T3SS, effector proteins and YadA, was included as a negative control because we surmised that Vn binding is pYV dependent. YadA protein levels were analyzed by Western blot analysis in whole-cell lysates and are shown below the bar chart (1 representative Western blot is shown). RNA polymerase protein (RNA-Pol.) was used as a loading control. YadA $\mathrm{O}^{\mathrm{O} 36471 / 76}$ has a calculated molecular weight of approximately $141 \mathrm{kDa}$ (455 aa), YadA ${ }^{\mathrm{O}: 8} 8081$ of $132 \mathrm{kDa}(422 \mathrm{aa})$, $\mathrm{YadA}^{\mathrm{O}: 8 \mathrm{WA}-314}$ of $132 \mathrm{kDa}(422 \mathrm{aa}), \mathrm{YadA}^{\mathrm{O}: 9 \mathrm{E} 40}$ of $153 \mathrm{kDa}(487$ aa), YadA ${ }^{\text {YPII }}$ of $135 \mathrm{kDa}$ (434 aa) and UspA2H of approximately $272 \mathrm{kDa}(876 \mathrm{aa})$. b To test if strain-specific differences in the bind- ing of $\mathrm{Vn}$ are exclusive, we compared Vn binding levels to that of factor $\mathrm{H}$. In contrast to $\mathrm{Vn}$, factor $\mathrm{H}$ is bound in comparable amounts by all Yersinia strains tested, except for the negative control strain (O:9 E40 $\Delta \mathrm{pYV})$. The protein levels of YadA and the RNA polymerase as a loading control were analyzed by Western blots of whole-cell lysates and are shown below the bar chart (1 representative Western blot is shown). c Binding of serum-derived Vn to Ye O:9 E40 is dose dependent. Ye O:9 E40 and the pYVcured version thereof were incubated with increasing serum concentrations. Afterwards, cell surface-associated Vn was quantified by flow cytometry. d Ye O:9 E40 and the pYV-cured version thereof were incubated with increasing amounts of purified Vn. Afterwards, cell surface-associated Vn was quantified by flow cytometry. Binding of purified Vn to Ye O:9 E40 is dose-dependent. a-d Data are means \pm SD of at least 4 individual experiments. a, b The main $\mathrm{p}$ values were determined by one-way ANOVA. $\mathrm{p}<0.0001$. Multiple comparisons were performed by one-way ANOVA with Dunnett's multiple-comparisons test. c, d The $\mathrm{p}$ values were determined by Student's t test. The error bars denote the SD. ${ }^{*} \mathrm{p}<0.05,{ }^{* *} \mathrm{p}<0.01,{ }^{* * *} \mathrm{p}<0.001,{ }^{* * * *} \mathrm{p}<0.0001$. 
with $Y e$ ) [18]. Ye O:9 E40 was able to bind exceptionally high amounts of $\mathrm{Vn}$, which led to a mean fluorescence intensity of approximately 2.8 times higher than that measured with $M c$ RH4 (133.9 \pm 33.9 vs. $47.4 \pm 19.6)$. Yps also bound $\mathrm{Vn}$, but at concentrations comparable to that of the Mc RH4 positive control $(56.7 \pm 11.0$ vs. $47.4 \pm$ 19.6). Ye O:8 WA-314 and Ye O:3 6471/176 also bound $\mathrm{Vn}$, though to a lesser extent than Mc RH4 (approx. 54.1 or $70.7 \%$ of $M c$ RH4 signal). Ye O:8 8081 bound only residual amounts of $\mathrm{Vn}(6.7 \pm 0.6)$. Interestingly, the binding of $\mathrm{Vn}$ to $\mathrm{Ye} \mathrm{O}: 9 \mathrm{E} 40$ depended on the presence of the plasmid of Yersinia virulence (pYV) and was dose dependent (fig. 1c, d). In a plasmid-deficient strain (Ye O:9 E40 $\Delta$ pYV), Vn binding was almost abolished (6.2 \pm 2.8$)$. To test if the strain-specific binding pattern of $\mathrm{Vn}$ (O:9 E40 > YPIII > RH4 > O:3 > O:8 WA-314 > O:9 E40 $\Delta \mathrm{pYV}=\mathrm{O}: 8$ 8081) is exclusive in comparison to other serum factors, we also tested the binding of factor $\mathrm{H}$ (fig. 1b). Factor $\mathrm{H}$ has been shown to interact with several discontinuous stretches within the stalk domain of YadA $[20-22,58]$. Our data corroborate previous findings that the binding of factor $\mathrm{H}$ by Yersinia strains relies on the presence of YadA, but in contrast to $\mathrm{Vn}$, there is no significant difference in binding efficiency in the various serotypes tested. This indicates different mechanisms of binding of $\mathrm{Vn}$ and factor $\mathrm{H}$. Taken together, we found that $Y e$ O:9 E40 is able to bind high amounts of serumderived as well as purified $\mathrm{Vn}$, although only in the presence of the pYV plasmid, in a dose-dependent manner. In contrast, $Y e$ O:8 WA-314, 8081 and $Y e$ O:3 6471/76 are weak $\mathrm{Vn}$ binders, although they also carry the pYV plasmid. This partially substantiates earlier findings that YadA-dependent Vn binding is at least weak if not nonexistent for $Y e$ O:8 WA-314 in whole-cell adhesion assays under specific flow conditions [45].

\section{Binding of Vn Is YadA Dependent}

In order to assess whether YadA is the determinant for the binding of Vn to Yersinia, we used flow cytometry to compare Vn binding in a Ye O:9 E40 WT strain, a mutant deficient for YadA ( $\triangle$ YadA), a mutant deficient for the chromosome-encoded adhesin Inv $(\Delta \operatorname{Inv})$, the corresponding double mutant $(\Delta \operatorname{Inv} \Delta \operatorname{YadA} ; \Delta \Delta)$ and, again, the cured strain lacking the pYV plasmid ( $\Delta$ pYV) (fig. 2a, left panel). We used $M c$ RH4 as positive control and an $M c \Delta \mathrm{UspA} 2 \mathrm{H}$ [32] knockout strain as a negative control (fig. 2a, right panel). Our data indicate that the presence of YadA, but not of Inv, is decisive for the binding of $\mathrm{Vn}$ to $\mathrm{Ye} \mathrm{O}: 9 \mathrm{E} 40$. Thus, in contrast to $\mathrm{Ye}$ O:9 E40 WT or $\Delta \mathrm{Inv}, \mathrm{Vn}$ did not bind to $\Delta \mathrm{YadA}$, the
$\Delta \operatorname{Inv} \Delta$ YadA double mutant or the pYV-cured strain. We could corroborate these findings by blot overlay assays and Western blot (fig. 2b, c). Analysis of the influence of YadA and, more specifically, a distinct region within YadA of Yps for Vn binding revealed that in Yps, YadA is also the Vn binding determinant (fig. 2a, middle panel). Moreover, the deletion of 30 aa $(\Delta 53-83)$ corresponding to the uptake region in the head domain of $Y p s$ YadA $^{\text {YPIII }}$ abolishes Vn binding (fig. 2a, middle panel). Thus, our data demonstrate that YadA is essential for mediating $\mathrm{Vn}$ binding in $Y e$ and that a stretch of 31 aa within the head region of YadA ${ }^{\mathrm{YIII}}$ is decisive for the binding of $\mathrm{Vn}$ in Yps.

\section{A Specific Stretch within YadA Discerns Low-Affinity}

Binding from High-Affinity Binding of Vn

We found that $Y e$ expressing YadA derived from the O:8 WA-314 strain is a relatively weak binder compared to $Y e$ O:9 E40 (fig. 1a). Therefore, we aimed to determine if other strains also carry the uptake region and also what actually discerns $\mathrm{YadA}^{\mathrm{O}: 9 \mathrm{E} 40}$ from $\mathrm{YadA}^{\mathrm{O}: 8}$ and if this difference might be causative for the discriminative $\mathrm{Vn}$ binding behavior. The head domain of YadA ${ }^{\text {YPIII contains }}$ a stretch of sequence (uptake region) which is crucial for cell adhesion and efficient internalization of Yersinia via YadA [18]. This motif is absent in YadA of Ye O:8 but present in the Ye O:9 E40 strain (aa 56-88) (fig. 3a). It is rich in prolines and charged residues, suggesting an undefined loop structure (fig. 3b), inserted in a shorter loop that is not resolved in the crystal structure of the $Y e$ O:3 YadA head (PDB: 1P9H) [59].

To investigate whether this motif is present exclusively in Ye O:9 E40 or can be found also in other Yersinia strains and especially in strains isolated from clinical specimens, we carried out PCRs. We designed primers binding to rather conserved regions within the YadA sequence flanking that part of the head domain which comprises the uptake region (online suppl. fig. S1). The size of the PCR products allowed us to easily detect the presence of the uptake region. The predicted lengths of the YadA head fragments were $346 \mathrm{bp}$ ( $Y e$ O:3), $337 \mathrm{bp}$ ( $Y e$ O:8), 346 bp ( $Y e$ O:5,27), 451 bp ( $Y e$ O:9) and 442 bp (Yps YPIII). Strikingly, the additional stretch present in YadA of $Y e$ O:9 E40 and Yps YPIII was present in all tested clinical isolates of serotype O:9 but absent in all other strains (belonging to the indicted serotypes; fig. 3c) that we tested. Ye O:9 E40 $\Delta$ YadA and water control were included as negative controls (fig. $3 \mathrm{c}$, all strains depicted were also tested for Vn binding). Cell surface-associated Vn after incubation in HIS was quantified by flow cytometry
Mühlenkamp et al. 
(fig. 3d). Whereas all strains belonging to serotype O:9 (No. 08-14) and possessing the uptake region within YadA bound Vn in comparably high amounts as $\mathrm{Ye}$ E40 O:9, Ye strains of serotype O:3 (No. 01, 02, 03), O:8 (No. 04 ) and O:5,27 (No. 06, 07) turned out to be rather weak binders. Thus, we assume that the presence of the uptake region is the major determinant that allows binding of $\mathrm{Vn}$ and (at least in the strains we have tested) is present exclusively in the YadA of Ye strains of serotype O:9.

To test this hypothesis, we generated a YadA hybrid where we replaced the $\mathrm{N}$-terminus of the head domain of $\mathrm{YadA}^{\mathrm{O}: 8}$ by that of $\mathrm{YadA}^{\mathrm{O}: 9 \mathrm{E} 40}$ (including the uptake region) and a YadA ${ }^{\mathrm{O} 9} \mathrm{E} 40$ deletion mutant lacking the uptake region (aa 56-88) (fig. 4a). We then compared $\mathrm{Vn}$ binding by flow cytometry. The strains $Y e$ O:9 $\Delta \mathrm{YadA}$ expressing $\mathrm{YadA}^{\mathrm{O}: 8 \mathrm{WA}-314}$ or $\mathrm{YadA}^{\mathrm{O}: 9 \mathrm{E} 40}$ were also included in this analysis. In addition, we used $Y e$ $\mathrm{O}: 8 \mathrm{WA}-314, \mathrm{Ye}$ O:9 E40 and $Y e \mathrm{O}: 9 \mathrm{E} 40 \Delta \mathrm{YadA}$ as controls (fig. 4b). Ectopic expression of $\mathrm{YadA}^{\mathrm{O}: 9 \mathrm{E} 40}$ was able to rescue Vn binding of Ye O:9 E40 $\Delta$ YadA. This was also true for the O:9/O:8 hybrid YadA. Additionally, deletion of the uptake region from $\mathrm{YadA}^{\mathrm{O}: 9}$ led to significantly reduced Vn binding (fig. 4b). Our data show that the uptake region of $\mathrm{YadA}^{\mathrm{O}: 9 \mathrm{E} 40}$ significantly enhances

Fig. 2. Vn binding to $Y e$ is YadA dependent. a Left panel: a Ye O:9 E40 WT strain or strains carrying individual deletions for the adhesins Invasin $(\Delta \mathrm{Inv})$ or $\mathrm{YadA}(\Delta \mathrm{YadA})$ and a respective double knockout strain $(\Delta \Delta)$ as well as a virulence plasmid-cured strain $(\Delta \mathrm{pYV})$ were incubated with serum and washed, and then Vn binding was quantified by flow cytometry. Middle panel: Yps YPIII WT and corresponding strains lacking expression of $\Delta \mathrm{YadA}$ or expressing a YadA version lacking part of the head domain $(\Delta 53-$ 83) were included as controls. Right panel: an Mc WT strain known to bind $\mathrm{Vn}$ via the surface adhesin UspA2 and a corresponding strain lacking expression of UspA2 ( $\triangle \mathrm{U} s \mathrm{sp} 2)$ were included as positive and negative controls. YadA protein levels were analyzed by Western blot analysis of whole-cell lysates and are shown below the bar chart (1 representative blot is shown). $\mathbf{b}$ A selection of the strains used in (a) was tested for Vn binding in a blot overlay assay. $\mathrm{Vn}$ and YadA were detected on the identical blot with specific antibodies and differently labeled secondary antibodies (emission maximum at 680 and $800 \mathrm{~nm}$, respectively) simultaneously. $\mathrm{Vn}$ is bound only in the presence of YadA $(Y e)$ or UspA2, respectively. c In a direct binding assay, essentially performed as in a, Vn can be detected at the expected molecular weight ( 65 and $75 \mathrm{kDa}$ ) by Western blot only in those Ye strains expressing YadA. Data are means \pm SD of at least 4 individual experiments (a) or $1 / 3$ representative experiments is shown $(\mathbf{b}, \mathbf{c})$. The main $\mathrm{p}$ value was determined by one-way ANOVA $(\mathbf{a}: \mathrm{p}<0.0001)$. Multiple comparisons were performed by one-way ANOVA with Dunnett's multiplecomparisons test. The error bars denote the SD. ${ }^{* *} \mathrm{p}<0.01$, $* * * * \mathrm{p}>0.0001$.

YadA-Mediated Interaction of $Y e$ with Vitronectin

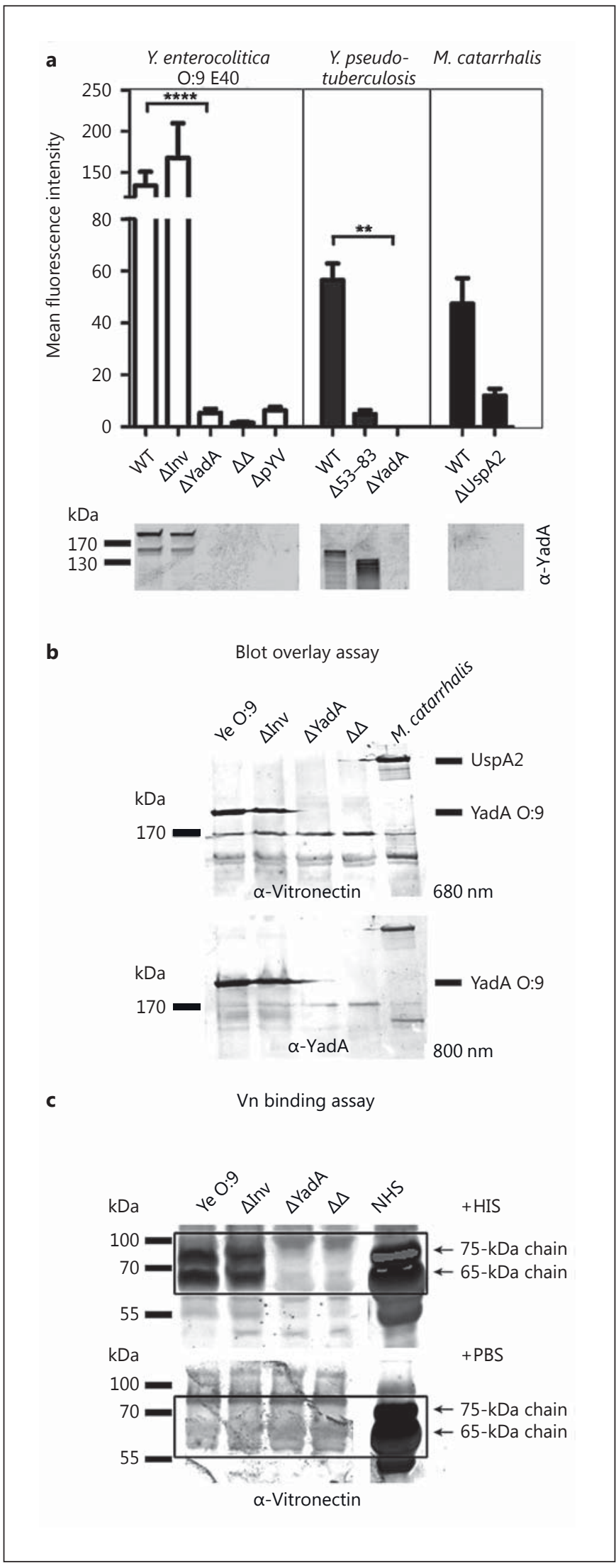

J Innate Immun 2017;9:33-51

DOI: $10.1159 / 000449200$ 


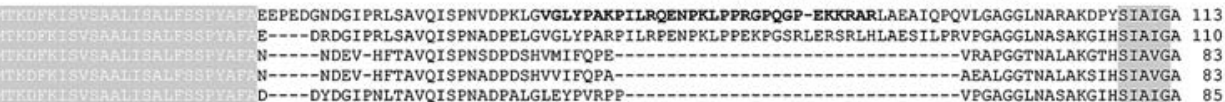

Yps YPIII

Ye $0: 9 \quad 840$

$\begin{array}{lll}\text { Ye } & 0: 8 & \text { WA- } 314 \\ \text { Ye } 0: 8 & 8081\end{array}$

TAEAAKPAAVAVGSGSIATGVNSVAIGPLSKALGDSAVTYGASSTAQKDGVAIGARASASDTGVAVGFNSKVDAQNSVAIGHSSHVAADHGYSIAIGDHSKTDRENSVSIGHE 226

TAEAAKEAAVAVGAGTIATGVNSVAIGPLSKALGDSAVTYGAASTAQKDGVAIGARASTSDTGVAVGFNSKADAKNSVAIGHSSHVAVDHDYSIAIGDRSKTDRENSVSIGHE 223

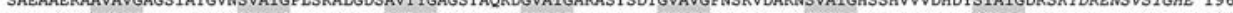
Se $0: 88081$ SAEAAKQAAVAVGAGSIATGVNSVAIGPLSKALGDSAVTYGAASTAQKDGVAIGARAFTSDTGVAVGFNSKVDAKNSVAIGHSSHVAVDHDYSIAIGDRSKTDRENSVSIGHE 196

b $\quad$ Ye $0: 36471 / 76$ Ye O:8 8081

Ye 0:8 WA-314
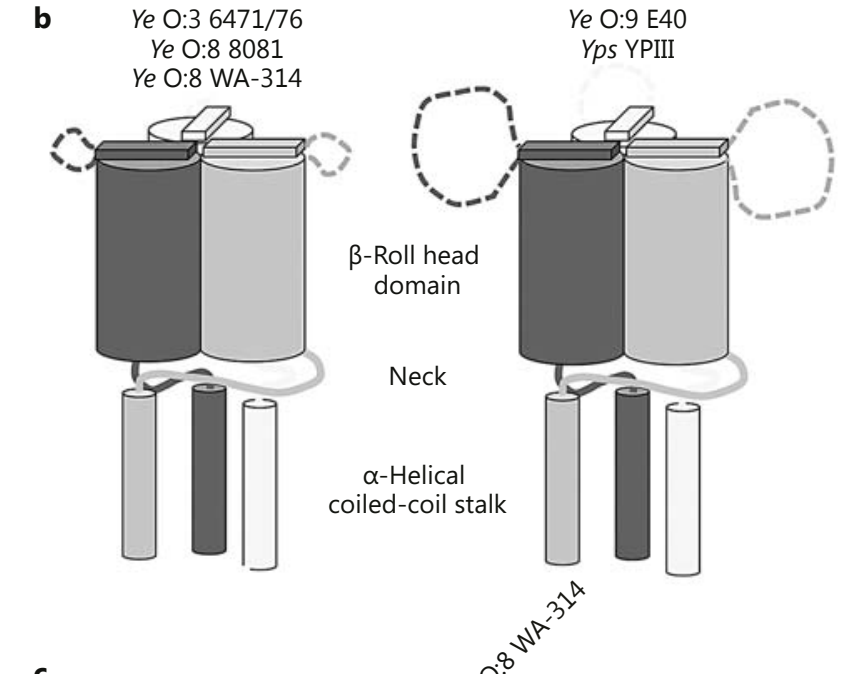

M $\begin{array}{lll}01 & 02 \quad 03\end{array}$

04

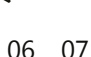

08

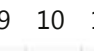

$\begin{array}{ll}0: 8 & 0: 5,27\end{array}$

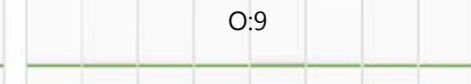

O:9

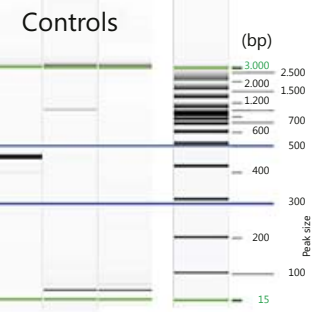

d
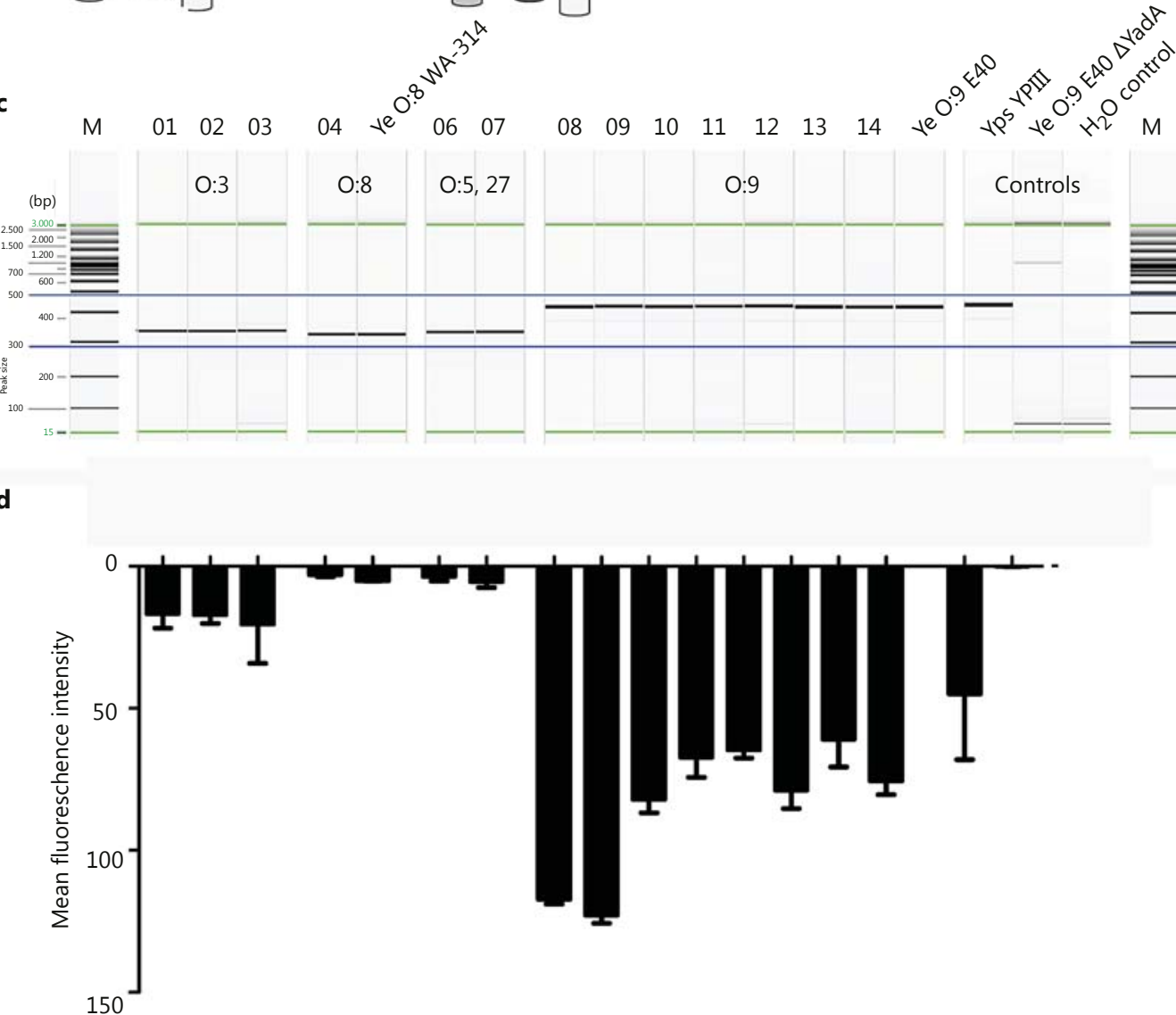

3

(For legend see next page.) 
recruitment of $\mathrm{Vn}$. Of note, a sequence alignment of YadA from different Yersinia strains also revealed insertions in the stalk regions of $Y e \operatorname{YadA}^{\mathrm{O}: 9 \mathrm{E} 40}$ and $\mathrm{YadA}^{\mathrm{O}: 36471 / 76}$ that are not found in $\mathrm{YadA}^{\mathrm{YPIII}}$ and $\mathrm{YadA}^{0: 8}$ WA-314 (online suppl. fig. S2). However, these regions show no clear association with $\mathrm{Vn}$ or factor $\mathrm{H}$ binding (fig. 1a). Finally, we wanted to assess whether cofactors expressed by Yersiniae are necessary or if YadA containing the uptake region alone is sufficient to mediate efficient binding of $\mathrm{Vn}$. We tested $\mathrm{Vn}$ binding of $E$. coli omp2 [60] which ectopically expressed the YadA version described above (online suppl. fig. S3). We found that expression of $\operatorname{YadA}^{\mathrm{O}: 9}$ or the hybrid $\operatorname{YadA}^{\mathrm{O}: 9 / \mathrm{O}: 8}$ is sufficient to mediate the binding of $\mathrm{Vn}$. Thus, we conclude that the decisive factor for Vn binding is YadA comprising the uptake region.

\section{Vn Interacts with YadA via Its C-Terminal HBD-3}

Previous work with $\mathrm{Mc}$ and $\mathrm{Hi}$ revealed HBD-3 as the decisive part of $\mathrm{Vn}$ for interaction with UspA2 or Hsf [34, 35]. Therefore, we wanted to know if this domain might also mediate the interaction of $\mathrm{Vn}$ with YadA. In order to test this, we first analyzed whether heparin might block the binding of $Y e$ to Vn by occupying the HBDs. This would be a clear indicator of the involvement of one of the HBDs in the interaction with YadA. Coverslips were coated with Vn and then incubated with Ye O:9 E40, expressing enhanced green fluorescent protein for easier detection of binding, either in the presence or absence of heparin. Thereafter, coverslips were washed, fixed, mounted and analyzed by fluorescence microscopy (fig. 5a). Our results demonstrate that, in the presence of heparin, the binding of bacteria to Vn-coated coverslips is significantly reduced. Therefore, we conclude that at

Fig. 3. A specific region in the YadA head domain is decisive for efficient binding of Vn. a Alignment of the head of various YadA variants. White letters on gray background: signal peptide. Black letters on gray background: the canonical 'SVAIG' head repeats of YadA. Italics: the neck region that links the head to the coiled-coil stalk of YadA. The insertion of Yps originally proposed by Heise and Dersch [18] is displayed in bold, and is slightly shifted towards the N-terminus of YadA. The dashed line on top shows the corrected position of the insertion, based on improved alignments and the structure of the YadA head from Ye O:3, where the short insertion is not resolved (underlined region). This and the unusually high number of prolines in this region suggest that it is not structured. The long version of the insertion carries a strongly positive net charge ( +5 for Yps YPIII, +4 for the Ye O:9 E40), which probably plays a role in binding to fibronectin and Vn. b Sche- least one of the HBDs is involved in mediating the binding of $\mathrm{Vn}$ to $\mathrm{YadA}^{0: 9} \mathrm{E} 40$.

To locate the sites within $\mathrm{Vn}$ that actually determine YadA binding, we used a set of recombinant Vn fragments (fig. 5b). These fragments essentially comprise Cterminal-truncated $\mathrm{Vn}$ molecules as well as deletion mutants lacking parts of HBD-3 (comprising aa 348-361) or adjacent regions. All fragments were tested for appropriate quality (online suppl. fig. S4). Our binding assay (fig. 5c) demonstrates that the fragments Vn 80-396, 80$379,80-373$ and $80-363$ are efficiently bound by Ye O:9 E40. However, further C-terminal truncation, comprising either parts of or the entire HBD-3 (80-353, 80-339), led to a reduction of binding. Fragments lacking the entire HBD-3 plus the adjacent N-terminal region (80-330, 80-229) bound only weakly to $\mathrm{Ye}$ O:9 E40 (fig. 5c). Thus, we assume that not only HBD-3 but also the adjacent $\mathrm{N}$ and especially the C-terminal approximately $10-20$ aa are important for a stable interaction of Vn with $Y e$ O:9 E40. These findings are in agreement with the fact that a Vn molecule lacking the C-terminal part of HBD-3 plus the adjacent C-terminal region $(\Delta 352-374)$ is also impaired when binding to $Y e$ O:9 E40 whereas deletion of either only part of HBD-3 ( $\triangle 352-362)$ or only the adjacent Cterminal region $(\Delta 362-374)$ does not significantly influence binding. In conclusion, aa 331-363 are decisive for the stable interaction of $\mathrm{Vn}$ with $\mathrm{Ye}$ O:9 E40.

\section{Vn Is Functionally Active and Inhibits the Terminal}

Pathway when Bound to the Surface of Ye

Besides modulating the adhesive properties of pathogens, $\mathrm{Vn}$ regulates the terminal complement pathway and blocks TCC formation. In order to test if $\mathrm{Vn}$ bound to $\mathrm{Ye}$ is functionally active and inhibits the terminal comple- matic view of the differences in the YadA heads. The Yps YPIII and $\mathrm{Ye}$ O:9 E40 variants have long insertions in an unstructured loop region close to the $\mathrm{N}$-terminus of the head. c PCR products comprising the YadA head region of $Y e$ O:8 WA-314, Ye O:9 E40 with and without YadA, Yps YPIII and clinical isolates derived from fecal samples ( $Y e$ O:3, No. 01-03; Ye O:8, No. 04; Ye O:5,27, No. 06-07 and Ye O:9, No. 08-12) or blood (No. 13) were separated by capillary gel electrophoresis. The predicted length of PCR products was as follows: Ye O:3 $346 \mathrm{bp}$; Ye O:8 $337 \mathrm{bp}$; Ye O:9 $451 \mathrm{bp}$; Ye O:5,27 $346 \mathrm{bp}$, and Yps YPIII $442 \mathrm{bp}$. Water control and a YadA-deficient strain were included as negative controls. d The strains shown in c were tested for Vn binding. Cell surface-associated $\mathrm{Vn}$ after incubation in HIS was quantified by flow cytometry. One of 3 representative experiments is shown. 
ment pathway, we assayed C5b-9 deposition in the presence of $\mathrm{Vn}$ bound to intact bacteria. To this end, $\mathrm{Ye} \mathrm{O}: 9$ E40 was preincubated with Vn or C4BP followed by the addition of C5b-6, C7, C8 and C9. C5b-9 deposition was determined by using an anti-C $5 \mathrm{~b}-9 \mathrm{mAb}$ and flow cytometry.

We clearly demonstrate that Vn bound to the surface of Ye O:9 E40 was functionally active and inhibited C5b-9 deposition in a dose-dependent manner (fig. 6a, b). Vn $(50 \mu \mathrm{g} / \mathrm{ml})$ inhibited C5b-9 deposition by $61 \%$. $\mathrm{C} 4 \mathrm{BP}$, the $\mathrm{C} 3$ convertase inhibitor of the classical/lectin pathways, did not influence the C5b-9 deposition and thus the terminal pathway. From this, we conclude that $V n$ when bound to intact $Y e$ is functionally active and inhibits the terminal complement pathway and C5b-9 deposition.

\section{Binding of Vn Decreases the Susceptibility to} Complement-Mediated Killing by Human Serum

YadA-mediated serum resistance is an important virulence trait of $Y e[21,61,62]$. To analyze the importance of $\mathrm{Vn}$ binding for preventing complement-mediated killing, we performed serum killing assays. We incubated $Y e$ O:9 E40, the corresponding YadA-deficient mutant ( $\triangle \mathrm{YadA}$ ) and $Y e$ O:8 WA-314 in NHS (fig. 7a, 'control strains'). Their survival was calculated as the survival percentage, taking the bacterial counts obtained with samples incubated in HIS as $100 \%$. Our data show that $Y e$ O:9 E40 - a strong Vn binder - is resistant to complement-mediated killing (\% survival in NHS compared to HIS $119.1 \pm 40.39$ ) whereas the Ye O:9 E40 $\triangle$ YadA mutant strain was highly susceptible for killing by the complement system $(16.74 \pm 9.83)$. Compared to $Y e$ O:9 E40, the weak Vn binder Ye O:8 WA-314 was significantly more susceptible to complement-mediated killing $(39.21 \pm 7.11)$ than $Y e$ O:9 E40 (fig. 7a). Furthermore, we also tested $Y e$ O:9 E40 $\Delta \Delta$ expressing either $\mathrm{YadA}^{\mathrm{O}: 9}, \mathrm{YadA}^{\mathrm{O}: 9 / \mathrm{O}: 8}, \mathrm{YadA}^{\mathrm{O}: 9 \Delta \text { uptake region }}$ or $\mathrm{YadA}^{\mathrm{O}: 9}$ for serum resistance. We found that the expression of $\mathrm{YadA}^{\mathrm{O}: 9}(103.6 \pm 3.42)$ and also of the O:9/O:8 hybrid YadA $(105.7 \pm 27,56)$ conferred serum resistance comparable to that of the $Y e$ O:9 E40 WT strain. In contrast, the serum survival was significantly reduced upon the expression of $\operatorname{YadA}^{\mathrm{O}: 8}(48.55 \pm 9.36)$. Compared to all these strains, a strain expressing the YadA O:9 lacking the uptake region showed the greatest sensitivity towards serum treatment $(16.8 \pm 7.57)$. These data clearly indicate that the YadA-dependent binding of Vn plays an important role in preventing the lysis of $Y e$ by the complement system. a

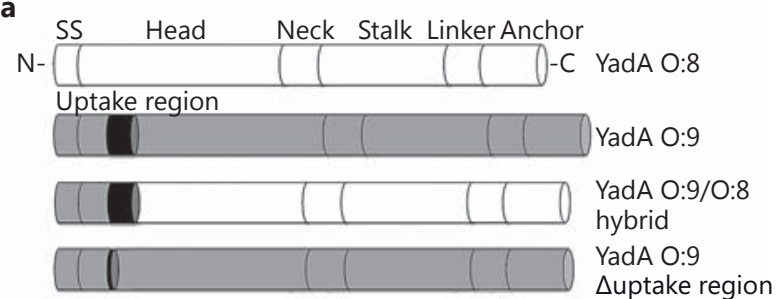

b

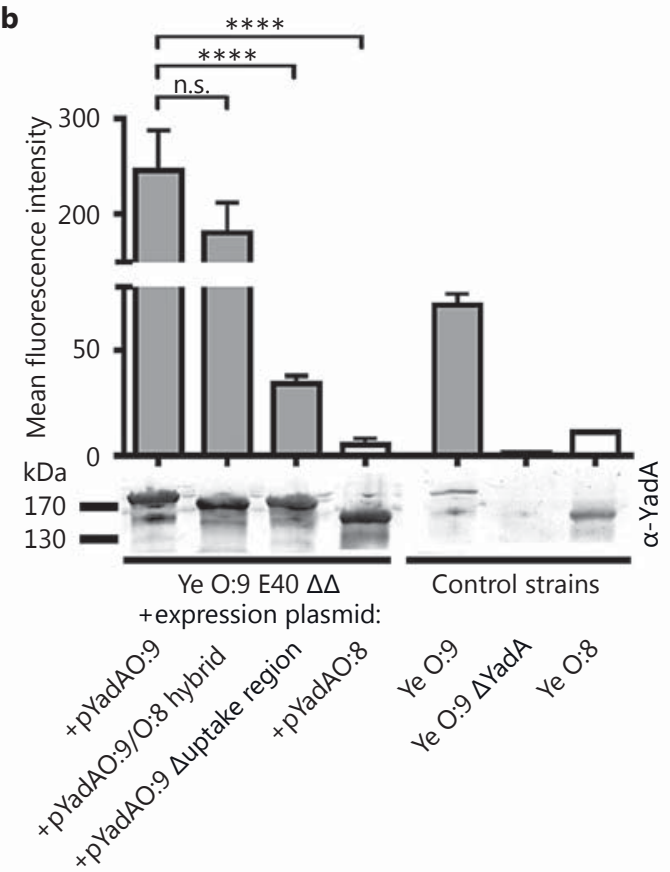

Fig. 4. The uptake region is decisive for YadA-mediated Vn binding. Schematic representation of different $Y$ adA versions that were expressed from a plasmid in $Y e$ O:9 E40 and analyzed for $\mathrm{Vn}$ binding capacity. The YadA versions tested comprise $\mathrm{YadA}^{\mathrm{O}: 8}$, YadA $^{\mathrm{O}: 9}$, a YadA ${ }^{\mathrm{O}: 9 / 0: 8}$ hybrid consisting of the O:9 head domain fused to the corresponding $Y e$ O:8 head/stalk and membrane anchor domain (details: Material and Methods) and $Y e \mathrm{YadA}^{\mathrm{O}: 9}$ with the uptake region deleted ( $\Delta$ uptake region). b Flow cytometry analysis of $\mathrm{Vn}$ binding to different $Y e$ strains carrying plasmids for inducible expression of the YadA versions depicted in a. As control strains, we used Ye strains expressing WT YadA from the endogenous pYV plasmid ( $Y e$ O:9 = positive control, $Y e$ O:8) and a $Y e$ O:9 E40 YadA-deficient strain ( $Y e$ O:9 $\Delta \mathrm{YadA}=$ negative control). YadA protein levels were analyzed by Western blot analysis in whole-cell lysates and are shown below the bar chart. Data are means \pm SD of at least 3 individual experiments (flow cytometry), or $1 / 3$ representative experiments is shown (Western blot). The main $p$ value was determined by one-way ANOVA (b, flow cytometry: $\mathrm{p}<0.0001$ ). Multiple comparisons were performed by oneway ANOVA with Dunnett's multiple-comparisons test. The error bars denote the SD. ${ }^{* * * *} \mathrm{p}>0.0001$.
44

J Innate Immun 2017;9:33-51

DOI: $10.1159 / 000449200$
Mühlenkamp et al. 
Fig. 5. Vn interacts with YadA via its Cterminal HBD-3. a Adhesion of $Y e$ to Vncoated coverslips can be blocked by heparin. $\mathbf{b}$ Schematic representation of Vn, the C-terminal-truncated Vn molecules [54] and the $\mathrm{Vn}$ molecules carrying deletions within and adjacent to the HBD-3 [35] that were used for a direct binding assay. c Western blot of a binding assay of $Y e$ O:9 E40 with full-length $\mathrm{Vn}$ and all fragments depicted in b. Vn fragments appear in green; YadA, which was detected simultaneously, appears in yellow bands (trimer runs at approx. $200 \mathrm{kDa}$ ). d Flow cytometry analysis of $\mathrm{Vn}$ binding to $\mathrm{Ye}$ O:9 E40 with full-length $\mathrm{Vn}$ and all fragments depicted in b. Data are means \pm SD of at least 3 individual experiments (a, d), or $1 / 3$ representative experiments is shown. The $p$ value for the comparison with and without heparin was determined by Student's t test. The main $\mathrm{p}$ value was determined by oneway ANOVA (d: p < 0.0001). Multiple comparisons were performed by one-way ANOVA with Dunnett's multiple-comparisons test. The error bars denote the SD. $* \mathrm{p}<0.05,{ }^{* * *} \mathrm{p}<0.001$.

YadA-Mediated Interaction of $Y e$ with Vitronectin a
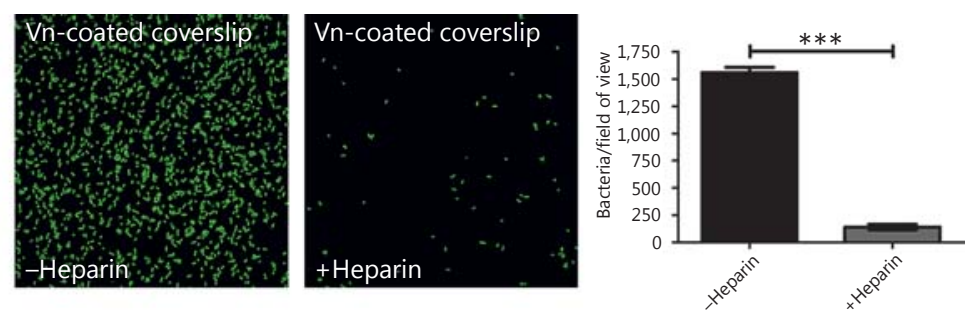

b

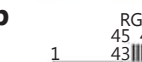

$R G D$
4547
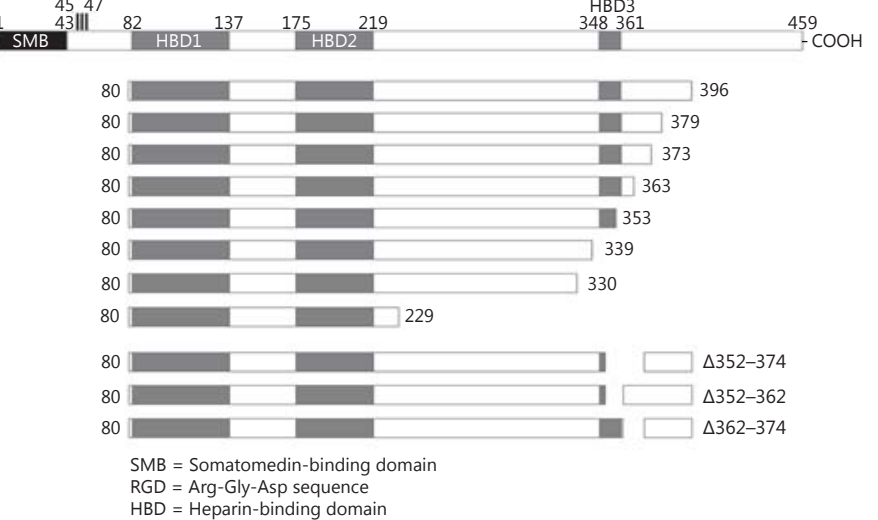

170
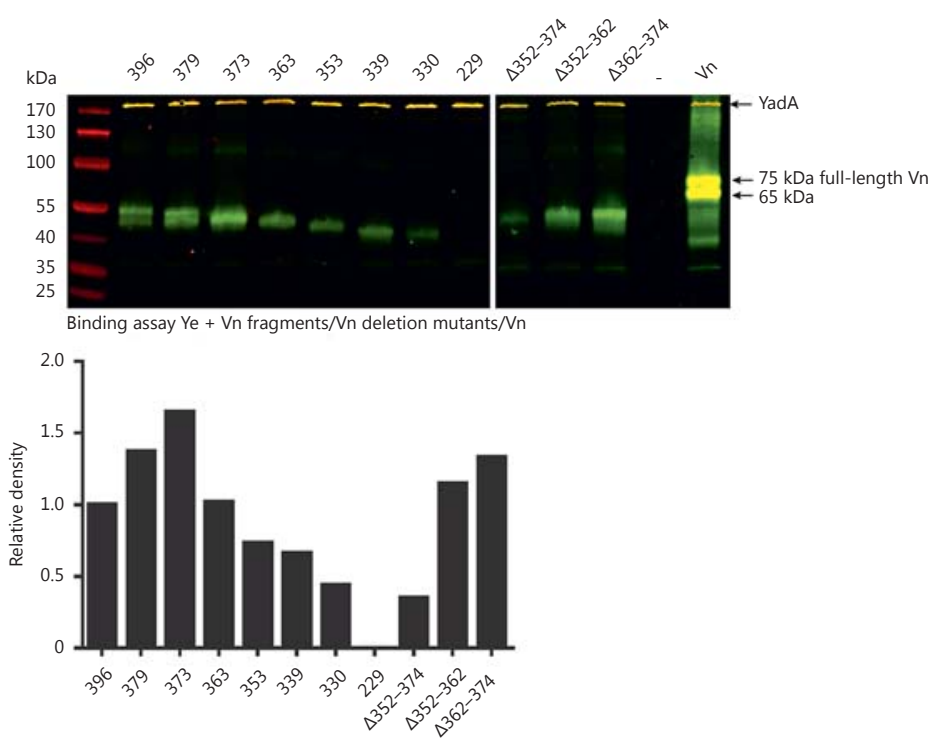

d

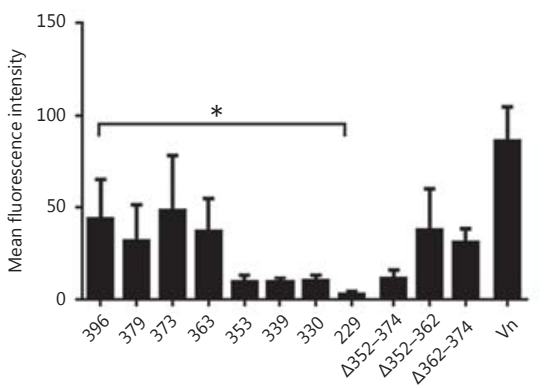




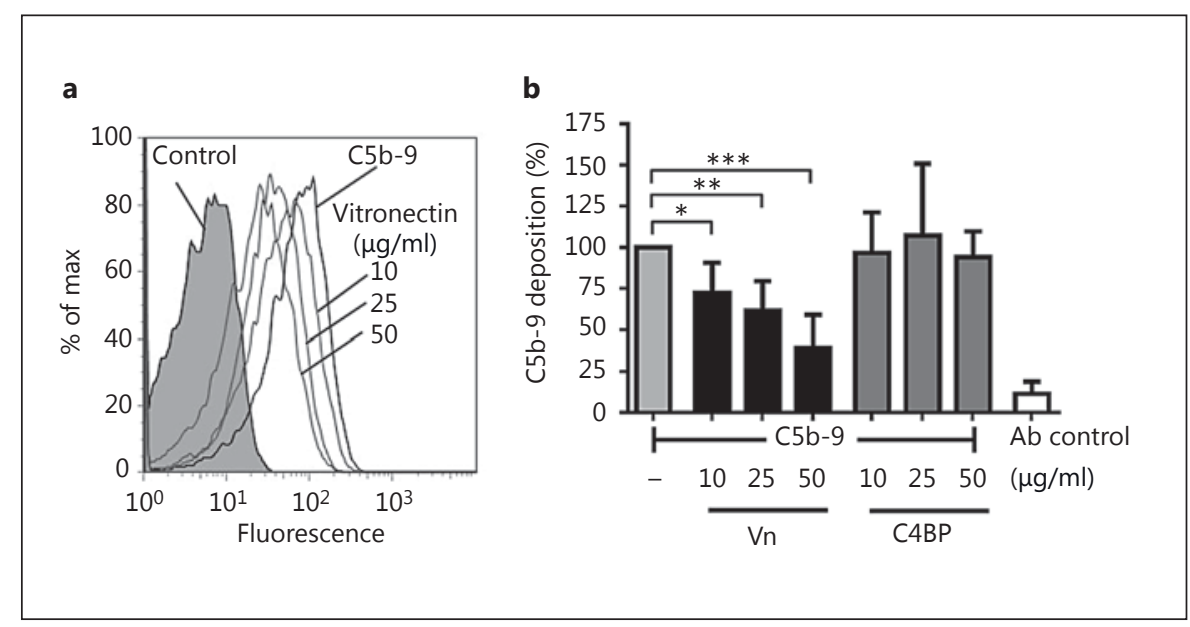

Fig. 6. $\mathrm{Vn}$ is functionally active and inhibits the terminal pathway when bound to the surface of $Y e$. a Histogram overlay of flow cytometry analyses of TCC formation (detected by formation of the neoepitope C5b-9) on the surface of $Y e$ O:9 E40 WT after preincubation of bacteria with PBS or different concentrations of $\mathrm{Vn}$ in PBS $(10,25$ and $50 \mu \mathrm{g} / \mathrm{ml})$. Preincubation with Vn reduces the amount of TCC that is formed. $\mathbf{b}$ Bar chart depicting C5b-9 deposition as percent of the amount of C5b-9 that was formed on the surface of bacteria preincubated with PBS only compared to bac-

Mice Deficient for Vn Expression Eliminate Ye More Rapidly in Short-Term Systemic Infection

It is known that YadA is decisive for the survival of $Y e$ upon contact with serum $[6,12]$. This is one reason why YadA-deficient strains of $Y e$ are avirulent in the mouse model [12]. However, the contribution of the YadA-dependent recruitment of $\mathrm{Vn}$ to the survival of $Y e$ in a mouse model has not been addressed so far. In order to test if the presence of $\mathrm{Vn}$ has an influence on the survival of $Y e$ in vivo, we infected $\mathrm{Vn}^{-/-}$and WT mice with $Y e$ O:9 E40, sacrificed the mice $30 \mathrm{~min}$ after infection and determined the bacterial burden in the blood. We found that the bacterial load in the blood was significantly reduced $\left(\log _{10}\right.$ CFU per gram of blood $=2.7 \pm 0.8$ ) for the $\mathrm{Vn}^{-/-}$mice compared to WT mice $\left(\log _{10} \mathrm{CFU}\right.$ per gram of blood $=$ $4.2 \pm 1.0$ ) (fig. 7b). In line with the reduction of C5b-9 deposition on $Y e$ by $\mathrm{Vn}$, these data would suggest that $\mathrm{Vn}$ protects $Y e$ from early killing in the blood stream.

Compared to YadA of $Y e$ O:9 E40, the YadA of Ye O:8 WA-314 shows a low Vn-binding capacity. Therefore, we hypothesized that due to this low Vn-binding capacity and in contrast to our findings with $Y e \mathrm{O}: 9 \mathrm{E} 40$, the availability of Vn should only marginally impact the outcome of an early bloodstream infection with the $Y e$ O:8 WA314 strain. However, since the $Y e$ O:9 and O:8 strains ex- teria preincubated with either Vn or C4BP at different concentrations $(10,25$ and $50 \mu \mathrm{g} / \mathrm{ml})$. Vn, but not C4BP, is able to reduce the formation of C5b-9. Antibody (Ab) control indicates background signal that was obtained using secondary antibody only for detection. Data are means \pm SD of at least 3 individual experiments. The main $p$ value was determined by one-way ANOVA ( $b$ : $p<0.001)$. Multiple comparisons were performed by one-way ANOVA with Dunnett's multiple-comparisons test. The error bars denote the SD. ${ }^{*} \mathrm{p}<0.05 ;{ }^{* *} \mathrm{p}<0.01,{ }^{* * *} \mathrm{p}<0.001$.

hibit additional differences with regard to sequence and also virulence mechanisms [63-66], this experiment may not solve the question of whether the uptake region actually contributes to better clearance of infection by mediating more efficient binding of $\mathrm{Vn}$ specifically. Therefore, we used a slightly different approach. To clearly assess the role of the uptake region and to exclude other differences between the $Y e$ O:8 and the $Y e$ O:9 strain tampering with the result of our experiments, we infected mice with $Y e$ harboring $\mathrm{pYadA}^{\mathrm{O}: 9 / 8 \text { hybrid }}$ or $\mathrm{pYadA}^{\mathrm{O}: 8}$ in the same strain background ( $Y e$ O:9 E40 $\Delta \Delta$ ). The basic sequence of the YadA of these strains is identical, with the exception of the part encoding the uptake region. Surprisingly, the infection of C57BL/6 WT or $\mathrm{Vn}^{-1-}$ mice with $\mathrm{Ye} \mathrm{O}: 9 \mathrm{E} 40 \Delta \Delta$ + pASK-IBA4c_yadAO:8 led to a small but significant difference in bacterial counts (online suppl. fig. S5A; 5.9 $\pm 0.3 \log _{10}$ CFU per gram of blood in WT mice vs. $6.4 \pm$ 0.3 in $\mathrm{Vn}^{-/-}$mice). As observed previously with Ye O:9 E40, infection with $Y e$ O:9 E40 $\Delta \Delta$ harboring pASKIBA4c_yadAO:9/O:8 hybrid revealed a significantly reduced bacterial load in the blood for the $\mathrm{Vn}^{-/-}$mice $\left(\log _{10}\right.$ CFU per gram of blood $=4.9 \pm 0.2)$ compared to WT mice $\left(\log _{10}\right.$ CFU per gram of blood $\left.=5.5 \pm 0.2\right)$ (online suppl. fig. S5B). This leads to the assumption that the binding of Vn to different regions of YadA may have various impli-
46

J Innate Immun 2017;9:33-51 DOI: $10.1159 / 000449200$
Mühlenkamp et al. 


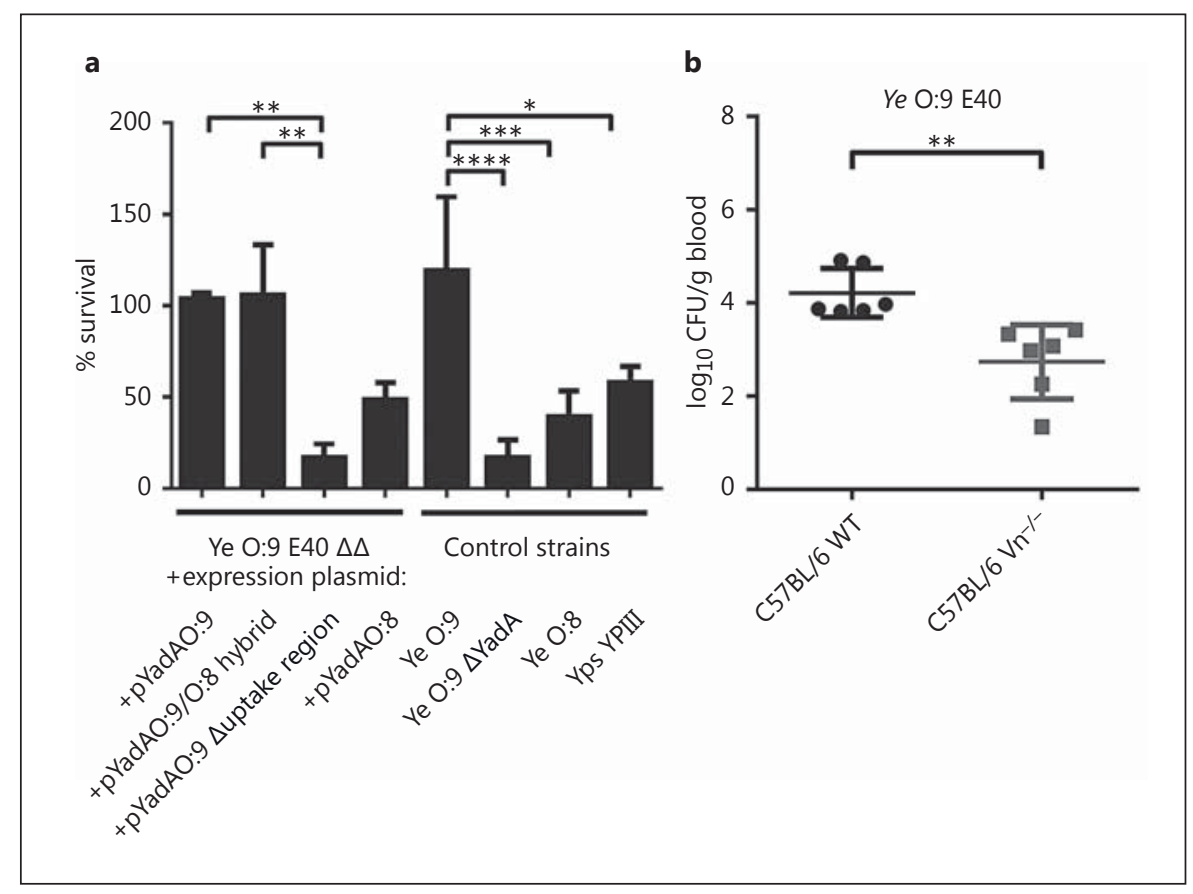

Fig. 7. Ye O:9 E40 is resistant to complement-mediated killing in vitro, and in an in vivo serum killing assay, $Y e$ is more efficiently eliminated in the absence of $\mathrm{Vn}$. a An in vitro serum killing assay using Ye O:9 E40, Ye O:9 E40 $\Delta \mathrm{YadA}, Y e$ O:8 WA-314, Yps YPII, Ye O:9 E40 $\Delta \Delta+$ pASK-IBA4C_yadAO:8, Ye O:9 E40 $\Delta \Delta+$ pASKIBA4C_yadAO:9, Ye O:9 E40 $\Delta \Delta+$ pASK-IBA4C_yadAO:9/O:8 hybrid, Ye O:9 E40 $\Delta \Delta+$ pASK-IBA4C_yadAO:9 $\Delta$ uptake region. The serum bactericidal effect was calculated as the survival percentage. $\mathbf{b} \mathrm{WT}$ and $\mathrm{Vn}^{-/-}$mice were infected intravenously with $1 \times 10^{7}$ Ye O:9 E40 for $30 \mathrm{~min}$. After that, the mice were killed, and blood was withdrawn and plated on selective agar plates. CFU were determined by counting colonies the next day, shown as $\log _{10}$ CFU per gram of blood. a Data are means \pm SD of at least 3 individual experiments. The main $\mathrm{p}$ value was determined by one-way ANOVA ( $p<0.0001)$. Multiple comparisons were performed by one-way ANOVA with Dunnett's multiple-comparisons test. b The $\mathrm{p}$ value for the comparison of $\mathrm{C} 57 \mathrm{BL} / 6$ and $\mathrm{Vn}^{-1-}$ mice was determined by Student's t test. The horizontal lines denote the mean and the error bars denote the SD. ${ }^{*} \mathrm{p}<0.05$; ${ }^{* *} \mathrm{p}<0.01$, *** $\mathrm{p}<0.001, * * * * \mathrm{p}>0.0001(\mathrm{n}=6)$. cations for YadA function. While binding of $\mathrm{Vn}$ to the uptake region seems to increase virulence, binding of $\mathrm{Vn}$ to other regions of YadA might also reduce virulence.

\section{Discussion}

Complement inhibitor recruitment by bacterial cell surface proteins and adhesins is an important virulence mechanism used by many pathogens. Accordingly, several complement regulators (factor $\mathrm{H}$, factor $\mathrm{H}$-like protein-1 and C4BP) and complement proteins (C3b and iC3b) have been identified that interact with the Gramnegative enteropathogen $\mathrm{Ye}$ [19-22, 61, 62, 67]. Here, we describe a novel mechanism that contributes to Ye complement resistance and the overall virulence of $Y e$. We show that the TAA YadA of different Yersinia species binds Vn and demonstrate that a part of the YadA head domain of $\mathrm{YadA}^{\mathrm{O}: 9} \mathrm{E} 40$ comprising aa 56-88 binds $\mathrm{Vn}$ with high efficiency. Recruitment of Vn to YadA led to the reduced surface formation and deposition of C5b-9 (TCC) and thus enhanced complement resistance. Moreover, Ye O:9 E40 was completely resistant to complement-mediated killing in human serum, in contrast to the YadA-deficient strain. In addition, it turned out that, in comparison to $Y e$ O:8 WA-314, Ye O:9 E40 is significantly more serum-resistant. Using Vn-deficient mice, we were also able to demonstrate the reduced survival of $Y e$ O:9 E40 in the absence of $\mathrm{Vn}$ in an in vivo serum killing assay. Thus, the binding of $\mathrm{Vn}$ to the surface of $Y e$ has a great impact on the interaction of $Y e$ with the host.

In our experiments, we found that different strains of $Y e$ and $Y p s$ bind Vn in a YadA-dependent manner but that different Yersinia strains exhibited divergent Vn- 
binding capacities. Previous studies with different $M c$ WT strains show that $M c$ also binds Vn with different affinities via UspA2 [30]. The N-terminus of the UspA2 head domain sequence displays 2 different conserved regions that may explain these Vn binding differences [68]. Furthermore, we show for the first time that $Y e$ strains of serotype O:9 - unlike all other Ye strains we tested - exhibit an additional stretch in their YadA head domain. These strains, and to a lesser extent Yps YPIII, showed high-affinity binding to $\mathrm{Vn}$ while other tested $Y e$ strains showed only low-affinity binding. Unfortunately, we were not able to correlate the ability to bind Vn and the pathogenic potential of clinical isolates due to the low frequency of $Y e$ infection (and thus available isolates) and the fact that systemic infection with $Y e$ happens only on rare occasions. The stretch in $\mathrm{YadA}^{\mathrm{O}: 9}$ is highly similar to the uptake region described for Yps YPIII [18], which is important for the ability of YadA to promote the invasion of $Y e$ into host cells. Yps binds preferentially to fibronectin, but has low affinity for laminin or collagen type I, which is in contrast to the ECM protein-binding capacity of $Y e$ which preferentially associates with collagen type I and laminin. This indicates that the uptake region may modulate the overall affinity to different ECM proteins. Sequence comparison of YadA ${ }^{\mathrm{O}: 9 \mathrm{E} 40}$ also revealed additional aa stretches in the YadA stalk domain, lacking in some other Ye strains. However, comparison of the Vnbinding capacity of different $Y e$ and $Y p s$ strains shows no clear indication that this region may also contribute to the differences in Vn binding, since YadA $\mathrm{O}^{\mathrm{O} 36471 / 76}$ has the same insertion in the stalk region. In contrast to $\mathrm{Vn}$ binding, the interaction with factor $\mathrm{H}$, which was shown to bind to the stalk region of YadA in $Y e$ and Yps strains, revealed no differences [20]. This indicates that the presence or absence of the uptake region modulates affinity to $\mathrm{Vn}$.

The site of interaction between $M c$ and $V n$ was mapped to the N-terminal residues 30-177 within UspA2 [34]. This region is located in the head domain of UspA2, which is similar to $\mathrm{YadA}^{\mathrm{O}: 9 \mathrm{E} 40}$. Our data show that subtle differences within the YadA protein sequence can significantly influence the protein interaction repertoire of $Y e$. The recruitment of such proteins to the surface of Ye may exert a significant influence on serum resistance and host cell interaction.

Localization of the Vn-binding domain within the YadA protein is a crucial step when analyzing the function of YadA in complement evasion. In contrast to complement regulator factor $\mathrm{H}$ or the complement component C3, which bind to the stalk domain of YadA [20], we found that $\mathrm{Vn}$ is bound via the YadA head domain. In $Y e$, the neutrophil-binding domain is located at the N-terminal part of YadA whereas the collagen-binding domain is located at the central and C-terminal part of the YadA head domain [59, 69-72]. The inhibition of Vn binding with heparin was already shown for $M c$ and $H i$. In both species, the interaction of $\mathrm{Vn}$ with UspA2 or Hsf was assigned to HBD-3 [34, 35]. In contrast, for $Y e \mathrm{O}: 9 \mathrm{E} 40$, not only HBD-3 but also the adjacent $\mathrm{N}$ - and C-terminal portions of $\mathrm{Vn}$ are decisive for the efficient interaction with YadA. We conclude that complement evasion of $Y e$ is not limited to interactions mediated by the stalk domain but can involve the head domain of YadA, depending on the strain in question. Furthermore, the uptake region in $Y e$ O:9 seems to provide a binding domain for $\mathrm{Vn}$ which strongly amplifies the binding of $\mathrm{Vn}$.

Previous studies showed that recruitment of $\mathrm{Vn}$ by $M c$ or $\mathrm{Hi}$ inhibits C5b-9 formation to block pore formation [27]. However, analyzing the TCC formation in Ye with purified complement proteins (C5b-6, C7, C8 and C9), we showed that bound Vn inhibits the deposition of C5b9 on the bacterial surface. Consequently, these data show that Vn bound to the bacterial surface via YadA is functionally active and inhibits the terminal pathway and thus contributes to complement resistance. Indeed, in in vitro serum killing assays, we showed that $Y e$ O:9 E40 is the strain that sustains treatment with serum most efficiently compared to Ye O:8 and Yps YPIII. In contrast, a YadAdeficient strain of $Y e$ O:9 E40 was susceptible to serum killing. Thus YadA-mediated binding of $\mathrm{Vn}$ in $\mathrm{Ye} \mathrm{O}: 9 \mathrm{E} 40$ is decisive for the success of serum treatment in vitro. The situation is different in $Y e$ O:8 WA-314. This strain is much more sensitive to serum treatment compared to $Y e$ $\mathrm{O}: 9$. We know that in $\mathrm{Ye} \mathrm{O}: 8$, serum resistance is mediated by the YadA-dependent recruitment of $\mathrm{C} 3 \mathrm{~b} / \mathrm{iC} 3 \mathrm{~b}$, factor $\mathrm{H}$ and C4BP $[21,33]$. As all these factors bind to YadA and, at least for C4BP, the binding site(s) within YadA is unknown, there might be competition for binding sites, and this might lead to the binding of low levels of Vn. Still, binding of all the other negative regulators of complement can mediate serum resistance to a certain extent. A decisive role of YadA for serum resistance of $Y p s$ YPIII is rather unlikely as it has been shown that Yps serum resistance occurs independently of the presence of a virulence plasmid (that encodes YadA [73]). The known mechanisms involved in the serum resistance of $Y p s$ are the binding of $\mathrm{C} 4 \mathrm{BP}$ and factor $\mathrm{H}$ via Ail $[74,75]$. Nevertheless, we have shown that Yps also binds Vn via YadA. We think that in this case the recruitment of Vn has a function other than mediating serum resistance and
48

J Innate Immun 2017;9:33-51

DOI: $10.1159 / 000449200$
Mühlenkamp et al. 
speculate that it might be involved in, for example, the modulation of host cell targeting [66] and interaction [24].

Consequently, this should also improve the survival of $\mathrm{Ye}$ in vivo. Indeed, the short-term infection of Vn-deficient mice with $Y e$ O:9 E40 revealed that Vn protects $Y e$ from being killed by the immune system. A short-term infection of mice was used to avoid (as far as possible) the action of other virulence mechanisms such as those provided by the T3SS. According to ex vivo measurements, the injection of Yops should efficiently show its action at later time points. Therefore, the short-term mouse experiments should predominantly reflect the impact of $\mathrm{Vn}$ on complement killing, as the complement system is activated within seconds after infection. Thus, the mouse infection experiments provide evidence that the inhibition of TCC formation by $\mathrm{V} n$ via binding to YadA indeed has biological relevance. These findings clearly demonstrate the importance of $\mathrm{Vn}$ binding to the uptake region for the pathogenicity of $Y e$. However, binding of $\mathrm{Vn}$ may also counteract YadA-mediated virulence, which is indicated by the slightly increased bacterial load after infection of $\mathrm{Vn}$-deficient mice with $\mathrm{Ye}$ O:9 E40 $\Delta \Delta$ expressing YadAO:8. We assume that the weak binding of Vn outside of the uptake region might interfere with the binding of other factors to YadA which are critical for YadA as a virulence factor. From an evolutionary point of view, the acquirement of the uptake region converts $\mathrm{Vn}$ from a factor protecting against infection to a factor mediating immune evasion.

Although individuals lacking terminal complement components are known to be more susceptible to N. meningitidis [76] but not especially to Ye infections, Vn binding is an important mechanism contributing to the over- all serum resistance of $Y e . Y e Y a d A$ interacts with a multitude of complement regulatory factors $(\mathrm{C} 4 \mathrm{bp}, \mathrm{C} 3 \mathrm{~b}$, $\mathrm{iC} 3 \mathrm{~b}$ and factor $\mathrm{H}$ ) that all contribute to serum resistance of $Y e$ in a true infection situation. These interactions in sum finally determine the success of $Y e$ within the host.

Taken together, our data present a novel mechanism of how YadA mediates immune evasion. By binding the HBD-3 domain of Vn, YadA containing the uptake region mediates the efficient inhibition of TCC formation and thus contributes to complement resistance and better survival of $Y e$. YadA is a multifunctional protein mediating complement resistance and also adhesion which, in turn, are critical for the subsequent injection of Yops into the host cells via the T3SS. Beyond bacteriolysis mediated by the assembly of the TCC, the even more important effect of Vn may be to modulate the interaction of $Y e$ with immune cells [66]. Further studies will now address how Vn may influence adhesion, invasion and Yop injection during mouse infection.

\section{Acknowledgments}

We thank T. Späth for excellent technical assistance, A. Fruth (Wernigerode), E. Carniel (Paris), M. Skurnik (Helsinki), J. Heesemann (Munich) and D. Villinger (Frankfurt) for providing the Y. enterocolitica clinical isolates, and P. Dersch (Braunschweig) for the gift of the Y.pseudotuberculosis strains as well as the antibody against Yps YadA. This work was funded by grants from the German Research Council (DFG) within the SFB 766 to M.S.S. and I.B.A., the Swedish Medical Research Council (grant No. K201557X-03163-43-4, http://www.vr.se) and the Anna and Edwin Berger Foundation to K.R., and it was supported by the Gender Equality Program E.05.00390 of the University Clinics Tübingen to M.S.S.

\section{References}

1 Bottone EJ: Yersinia enterocolitica: overview and epidemiologic correlates. Microbes Infect 1999; 1:323-333.

2 Cover TL, Aber RC: Yersinia enterocolitica. N Engl J Med 1989;321:16-24.

3 Isberg RR: Mammalian cell adhesion functions and cellular penetration of enteropathogenic Yersinia species. Mol Microbiol 1989;3: 1449-1453.

4 Isberg RR: Determinants for thermoinducible cell binding and plasmid-encoded cellular penetration detected in the absence of the Yersinia pseudotuberculosis invasin protein. Infect Immun 1989;57:1998-2005.
5 Miller VL, Falkow S: Evidence for two genetic loci in Yersinia enterocolitica that can promote invasion of epithelial cells. Infect Immun 1988;56:1242-1248.

6 El Tahir Y, Skurnik M: YadA, the multifaceted Yersinia adhesin. Int J Med Microbiol 2001;291:209-218.

7 Linke D, Riess T, Autenrieth IB, Lupas A, Kempf VA: Trimeric autotransporter adhesins: variable structure, common function. Trends Microbiol 2006; 14:264-270.

8 Hoiczyk E, Roggenkamp A, Reichenbecher M, Lupas A, Heesemann J: Structure and sequence analysis of Yersinia YadA and Moraxella UspAs reveal a novel class of adhesins. EMBO J 2000;19:5989-5999.
9 Mühlenkamp M, Oberhettinger P, Leo JC, Linke D, Schütz MS: Yersinia adhesin A (YadA) - Beauty and beast. Int J Med Microbiol 2015;305:252-258.

10 Szczesny P, Lupas A: Domain annotation of trimeric autotransporter adhesins - daTAA. Bioinformatics 2008;24:1251-1256.

11 Pepe JC, Wachtel MR, Wagar E, Miller VL: Pathogenesis of defined invasion mutants of Yersinia enterocolitica in a BALB/c mouse model of infection. Infect Immun 1995;63: 4837-4848.
YadA-Mediated Interaction of $Y e$ with Vitronectin
J Innate Immun 2017;9:33-51 DOI: $10.1159 / 000449200$ 
12 Schütz M, Weiss EM, Schindler M, Hallström T, Zipfel PF, Linke D, Autenrieth IB: Trimer stability of YadA is critical for virulence of Yersinia enterocolitica. Infect Immun 2010; 78:2677-2690.

13 Di Genaro MS, Waidmann M, Kramer U, Hitziger N, Bohn E, Autenrieth IB: Attenuated Yersinia enterocolitica mutant strains exhibit differential virulence in cytokine-deficient mice: implications for the development of novel live carrier vaccines. Infect Immun 2003;71:1804-1812.

14 Cornelis GR: Yersinia type III secretion: send in the effectors. J Cell Biol 2002;158:401-408.

15 Viboud GI, Bliska JB: Yersinia outer proteins: role in modulation of host cell signaling responses and pathogenesis. Ann Rev Microbiol 2005;59:69-89.

16 Hoyer-Hansen G, Behrendt N, Ploug M, Dano K, Preissner KT: The intact urokinase receptor is required for efficient vitronectin binding: receptor cleavage prevents ligand interaction. FEBS Lett 1997;420:79-85.

17 Eitel J, Dersch P: The YadA protein of Yersinia pseudotuberculosis mediates high-efficiency uptake into human cells under environmental conditions in which invasin is repressed. Infect Immun 2002;70:4880-4891.

18 Heise T, Dersch P: Identification of a domain in Yersinia virulence factor YadA that is crucial for extracellular matrix-specific cell adhesion and uptake. Proc Natl Acad Sci USA 2006; 103:3375-3380.

19 Biedzka-Sarek M, Jarva H, Hyytiäinen H, Meri S, Skurnik M: Characterization of complement factor $\mathrm{H}$ binding to Yersinia enterocolitica serotype O:3. Infect Immun 2008;76: 4100-4109.

20 Biedzka-Sarek M, Salmenlinna S, Gruber M, Lupas AN, Meri S, Skurnik M: Functional mapping of YadA- and Ail-mediated binding of human factor $\mathrm{H}$ to Yersinia enterocolitica serotype O:3. Infect Immun 2008; 76:50165027.

21 Schindler MK, Schütz MS, Mühlenkamp MC, Rooijakkers SH, Hallström T, Zipfel PF, Autenrieth IB: Yersinia enterocolitica YadA mediates complement evasion by recruitment and inactivation of $\mathrm{C} 3$ products. J Immunol 2012;189:4900-4908.

22 China B, Sory MP, N'Guyen BT, De Bruyere M, Cornelis GR: Role of the YadA protein in prevention of opsonization of Yersinia enterocolitica by C3b molecules. Infect Immun 1993;61:3129-3136.

23 Preissner KT: Structure and biological role of vitronectin. Annu Rev Cell Biol 1991;7:275310.

24 Boyd NA, Bradwell AR, Thompson RA: Quantitation of vitronectin in serum: evaluation of its usefulness in routine clinical practice. J Clin Pathol 1993;46:1042-1045.

25 Seiffert D, Crain K, Wagner NV, Loskutoff DJ: Vitronectin gene expression in vivo. Evidence for extrahepatic synthesis and acute phase regulation. J Biol Chem 1994;269: 19836-19842.
26 Liang OD, Rosenblatt S, Chhatwal GS, Preissner KT: Identification of novel heparin-binding domains of vitronectin. FEBS Lett 1997; 407:169-172.

27 Singh B, Su YC, Riesbeck K: Vitronectin in bacterial pathogenesis: a host protein used in complement escape and cellular invasion. Mol Microbiol 2010;78:545-560.

28 Milis L, Morris CA, Sheehan MC, Charlesworth JA, Pussell BA: Vitronectin-mediated inhibition of complement: evidence for different binding sites for C5b-7 and C9. Clin Exp Immunol 1993;92:114-119.

29 Sheehan M, Morris CA, Pussell BA, Charlesworth JA: Complement inhibition by human vitronectin involves non-heparin binding domains. Clin Exp Immunol 1995;101:136-141.

30 Attia AS, Ram S, Rice PA, Hansen EJ: Binding of vitronectin by the Moraxella catarrhalis UspA2 protein interferes with late stages of the complement cascade. Infect Immun 2006; 74:1597-1611.

31 Cole LE, Kawula TH, Toffer KL, Elkins C: The Haemophilus ducreyi serum resistance antigen DsrA confers attachment to human keratinocytes. Infect Immun 2002;70:6158-6165.

32 Hallström T, Trajkovska E, Forsgren A, Riesbeck K: Haemophilus influenzae surface fibrils contribute to serum resistance by interacting with vitronectin. J Immunol 2006;177: 430-436.

33 Leduc I, Olsen B, Elkins C: Localization of the domains of the Haemophilus ducreyi trimeric autotransporter DsrA involved in serum resistance and binding to the extracellular matrix proteins fibronectin and vitronectin. Infect Immun 2009;77:657-666.

34 Singh B, Blom AM, Unal C, Nilson B, Mörgelin M, Riesbeck K: Vitronectin binds to the head region of Moraxella catarrhalis ubiquitous surface protein A2 and confers complement-inhibitory activity. Mol Microbiol 2010;75:1426-1444.

35 Singh B, Su YC, Al-Jubair T, Mukherjee O, Hallström T, Mörgelin M, Blom AM, Riesbeck K: A fine-tuned interaction between trimeric autotransporter Haemophilus surface fibrils and vitronectin leads to serum resistance and adherence to respiratory epithelial cells. Infect Immun 2014;82:2378-2389.

36 Su YC, Jalalvand F, Mörgelin M, Blom AM, Singh B, Riesbeck K: Haemophilus influenzae acquires vitronectin via the ubiquitous protein $\mathrm{F}$ to subvert host innate immunity. Mol Microbiol 2013;87:1245-1266.

37 Sa ECC, Griffiths NJ, Virji M: Neisseria meningitidis Opc invasin binds to the sulphated tyrosines of activated vitronectin to attach to and invade human brain endothelial cells. PLoS Pathog 2010;6:e1000911.

38 Hill DJ, Griffiths NJ, Borodina E, Andreae CA, Sessions RB, Virji M: Identification and therapeutic potential of a vitronectin binding region of meningococcal MSF. PLoS One 2015;10:e124133.
39 Duensing TD, Putten JP: Vitronectin binds to the gonococcal adhesin OpaA through a glycosaminoglycan molecular bridge. Biochem J 1998;334(Pt 1):133-139.

40 Griffiths NJ, Hill DJ, Borodina E, Sessions RB, Devos NI, Feron CM, Poolman JT, Virji M: Meningococcal surface fibril (MSF) binds to activated vitronectin and inhibits the terminal complement pathway to increase serum resistance. Mol Microbiol 2011;82:1129-1149.

41 Bergmann S, Lang A, Rohde M, Agarwal V, Rennemeier C, Grashoff C, Preissner KT, Hammerschmidt S: Integrin-linked kinase is required for vitronectin-mediated internalization of Streptococcus pneumoniae by host cells. J Cell Sci 2009;122:256-267.

42 Kohler TP, Gisch N, Binsker U, Schlag M, Darm K, Völker U, Zähringer U, Hammerschmidt S: Repeating structures of the major staphylococcal autolysin are essential for the interaction with human thrombospondin 1 and vitronectin. J Biol Chem 2014;289:40704082.

43 Voss S, Hallström T, Saleh M, Burchhardt G Pribyl T, Singh B, Riesbeck K, Zipfel PF, Hammerschmidt S: The choline-binding protein PspC of Streptococcus pneumoniae interacts with the C-terminal heparin-binding domain of vitronectin. J Biol Chem 2013;288: 15614-15627.

44 Kohler S, Hallström T, Singh B, Riesbeck K, Spartà G, Zipfel PF, Hammerschmidt S: Binding of vitronectin and factor $\mathrm{H}$ to Hic contributes to immune evasion of Streptococcus pneumoniae serotype 3. Thromb Haemost 2015;113:125-142.

45 Müller NF, Kaiser PO, Linke D, Schwarz H, Riess T, Schäfer A, Eble JA, Kempf VA: Trimeric autotransporter adhesin-dependent adherence of Bartonella henselae, Bartonella quintana, and Yersinia enterocolitica to matrix components and endothelial cells under static and dynamic flow conditions. Infect Immun 2011;79:2544-2553.

46 Köberle M, Klein-Günther A, Schütz M, Fritz M, Berchtold S, Tolosa E, Autenrieth IB, Bohn E: Yersinia enterocolitica targets cells of the innate and adaptive immune system by injection of Yops in a mouse infection model. PLoS Pathog 2009;5:e1000551.

47 Keller B, Mühlenkamp M, Deuschle E, Siegfried A, Mössner S, Schade J, Griesinger T, Katava N, Braunsdorf C, Fehrenbacher B, Jimenez-Soto LF, Schaller M, Haas R, Genth H, Retta SF, Meyer H, Bottcher RT, Zent R, Schütz M, Autenrieth IB, Bohn E: Yersinia enterocolitica exploits different pathways to accomplish adhesion and toxin injection into host cells. Cell Microbiol 2015;17:1179-1204.

48 Skurnik M: Lack of correlation between the presence of plasmids and fimbriae in Yersinia enterocolitica and Yersinia pseudotuberculosis. J Appl Bacteriol 1984;56:355-363.

49 Portnoy DA, Moseley SL, Falkow S: Characterization of plasmids and plasmid-associated determinants of Yersinia enterocolitica pathogenesis. Infect Immun 1981;31:775-782. 
50 Strobel E, Heesemann J, Mayer G, Peters J, Müller-Weihrich S, Emmerling P: Bacteriological and serological findings in a further case of transfusion-mediated Yersinia enterocolitica sepsis. J Clin Microbiol 2000;38:27882790.

51 Bolin I, Norlander L, Wolf-Watz H: Temperature-inducible outer membrane protein of Yersinia pseudotuberculosis and Yersinia enterocolitica is associated with the virulence plasmid. Infect Immun 1982;37:506-512.

52 Mollenkvist A, Nordström T, Hallden C, Christensen JJ, Forsgren A, Riesbeck K: The Moraxella catarrhalis immunoglobulin Dbinding protein MID has conserved sequences and is regulated by a mechanism corresponding to phase variation. J Bacteriol 2003; 185:2285-2295.

53 Nordström T, Blom AM, Tan TT, Forsgren A, Riesbeck K: Ionic binding of $\mathrm{C} 3$ to the human pathogen Moraxella catarrhalis is a unique mechanism for combatting innate immunity. J Immunol 2005;175:3628-3636.

54 Singh B, Jalalvand F, Mörgelin M, Zipfel P, Blom AM, Riesbeck K: Haemophilus influenzae protein $\mathrm{E}$ recognizes the C-terminal domain of vitronectin and modulates the membrane attack complex. Mol Microbiol 2011; 81:80-98.

55 Edgar RC: MUSCLE: multiple sequence alignment with high accuracy and high throughput. Nucleic Acids Res 2004;32:17921797.

56 Lassmann T, Sonnhammer EL: Kalign - an accurate and fast multiple sequence alignment algorithm. BMC Bioinformatics 2005;6: 298.

57 Hallström T, Singh B, Resman F, Blom AM, Morgelin M, Riesbeck K: Haemophilus influenzae protein $\mathrm{E}$ binds to the extracellular matrix by concurrently interacting with laminin and vitronectin. J Infect Dis 2011;204:10651074.

58 Flügel A, Schulze-Koops H, Heesemann J, Kuhn K, Sorokin L, Burkhardt H, von der Mark K, Emmrich F: Interaction of enteropathogenic Yersinia enterocolitica with complex basement membranes and the extracellular matrix proteins collagen type IV, laminin-1 and -2, and nidogen/entactin. J Biol Chem 1994;269:29732-29738.
59 Nummelin H, Merckel MC, Leo JC, Lankinen H, Skurnik M, Goldman A: The Yersinia adhesin YadA collagen-binding domain structure is a novel left-handed parallel beta-roll. EMBO J 2004;23:701-711.

60 Prilipov A, Phale PS, Van Gelder P, Rosenbusch JP, Koebnik R: Coupling site-directed mutagenesis with high-level expression: large scale production of mutant porins from $E$. coli. FEMS Microbiol Lett 1998;163:65-72.

61 Pilz D, Vocke T, Heesemann J, Brade V: Mechanism of YadA-mediated serum resistance of Yersinia enterocolitica serotype O3. Infect Immun 1992;60:189-195.

62 Biedzka-Sarek M, Venho R, Skurnik M: Role of YadA, Ail, and lipopolysaccharide in serum resistance of Yersinia enterocolitica serotype O:3. Infect Immun 2005;73:2232-2244.

63 Dersch P, Isberg RR: An immunoglobulin superfamily-like domain unique to the Yersinia pseudotuberculosis invasin protein is required for stimulation of bacterial uptake via integrin receptors. Infect Immun 2000;68:2930-2938.

64 Deuschle E, Keller B, Siegfried A, Manncke B, Späth T, Köberle M, Drechsler-Hake D, Reber J, Böttcher RT, Autenrieth SE, Autenrieth IB, Bohn E, Schütz M: Role of betal integrins and bacterial adhesins for Yop injection into leukocytes in Yersinia enterocolitica systemic mouse infection. Int J Med Microbiol 2016; 306:77-88.

65 Duan R, Liang J, Shi G, Cui Z, Hai R, Wang P, Xiao Y, Li K, Qiu H, Gu W, Du X, Jing H, Wang X: Homology analysis of pathogenic Yersinia species Yersinia enterocolitica, Yersinia pseudotuberculosis, and Yersinia pestis based on multilocus sequence typing. J Clin Microbiol 2014;52:20-29.

66 Maldonado-Arocho FJ, Green C, Fisher ML, Paczosa MK, Mecsas J: Adhesins and host serum factors drive Yop translocation by Yersinia into professional phagocytes during animal infection. PLoS Pathog 2013;9:e1003415.

67 Kirjavainen V, Jarva H, Biedzka-Sarek M, Blom AM, Skurnik M, Meri S: Yersinia enterocolitica serum resistance proteins YadA and Ail bind the complement regulator $\mathrm{C} 4 \mathrm{~b}$ binding protein. PLoS Pathog 2008;4: e1000140.
68 Brooks MJ, Sedillo JL, Wagner N, Laurence CA, Wang W, Attia AS, Hansen EJ, Gray-Owen SD: Modular arrangement of allelic variants explains the divergence in Moraxella catarrhalis UspA protein function. Infect Immun 2008;76:5330-5340.

69 El Tahir Y, Kuusela P, Skurnik M: Functional mapping of the Yersinia enterocolitica adhesin YadA. Identification of eight NSVAIG-S motifs in the amino-terminal half of the protein involved in collagen binding. Mol Microbiol 2000;37:192-206.

70 Roggenkamp A, Neuberger HR, Flügel A, Schmoll T, Heesemann J: Substitution of two histidine residues in YadA protein of Yersinia enterocolitica abrogates collagen binding, cell adherence and mouse virulence. Mol Microbiol 1995;16:1207-1219.

71 Tamm A, Tarkkanen AM, Korhonen TK, Kuusela P, Toivanen P, Skurnik M: Hydrophobic domains affect the collagen-binding specificity and surface polymerization as well as the virulence potential of the YadA protein of Yersinia enterocolitica. Mol Microbiol 1993;10:995-1011.

72 Roggenkamp A, Ruckdeschel K, Leitritz L, Schmitt R, Heesemann J: Deletion of amino acids 29 to 81 in adhesion protein YadA of Yersinia enterocolitica serotype O:8 results in selective abrogation of adherence to neutrophils. Infect Immun 1996;64:2506-2514.

73 Perry RD, Brubaker RR: Vwa+ phenotype of Yersinia enterocolitica. Infect Immun 1983; 40:166-171.

74 Ho DK, Riva R, Kirjavainen V, Jarva H, Ginstrom E, Blom AM, Skurnik M, Meri S: Functional recruitment of the human complement inhibitor C4BP to Yersinia pseudotuberculosis outer membrane protein Ail. J Immunol 2012;188:4450-4459.

75 Ho DK, Riva R, Skurnik M, Meri S: The Yersinia pseudotuberculosis outer membrane protein Ail recruits the human complement regulatory protein factor $\mathrm{H}$. J Immunol 2012; 189:3593-3599.

76 Ram S, Lewis LA, Rice PA: Infections of people with complement deficiencies and patients who have undergone splenectomy. Clin Microbiol Rev 2010;23:740-780.
YadA-Mediated Interaction of $Y e$ with Vitronectin
J Innate Immun 2017;9:33-51

DOI: $10.1159 / 000449200$ 